\begin{abstract}
DENNIS, BRENT MOORMAN. Assisted Navigation of Large Information Spaces. (Under the direction of Dr. Christopher G. Healey)

With the advent of computers and more sophisticated electronics, scientists can now collect massive amounts of information. The increasing size and dimensionality of these datasets makes them challenging to visualize in an effective manner. Visualizations must show the global structure of spatial relationships within the dataset while simultaneously representing the local detail of each data element being visualized. Techniques in information visualization provide views of the dataset at multiple scales allowing the user to visualize large numbers of data elements. Unfortunately, these techniques often do not address the problems of visualizing multidimensional datasets. Multidimensional visualizations use color, texture, and other visual features to represent the values of multiple attributes at a single spatial location. However, these techniques do not address how to visualize large numbers of data elements.

Visualizations of datasets with large sizes and high dimensionalities are often forced to omit data elements from the current view. This thesis proposes to combine ideas from information and multidimensional visualization with a navigation assistant to help users identify and explore areas of interest within their data. The assistant identifies data elements that are potentially "interesting" to the user, clusters them into spatially coherent regions, and constructs underlying graph structures to connect the regions and the elements they contain. Using graph traversal algorithms, optimal viewpoint construction, and camera planning techniques, the navigation assistant builds informative animated tours of these regions. In this way, the assistant provides an effective tool for exploring a dataset.
\end{abstract}




\title{
Assisted Navigation of Large Information Spaces
}

by

\section{BRENT M. DENNIS}

\author{
A thesis submitted to the Graduate Faculty of \\ North Carolina State University \\ in partial fulfillment of the \\ requirements for the Degree of \\ Master of Science
}

\author{
Department of \\ COMPUTER SCIENCE \\ Raleigh, North Carolina
}

2002

APPROVED BY:
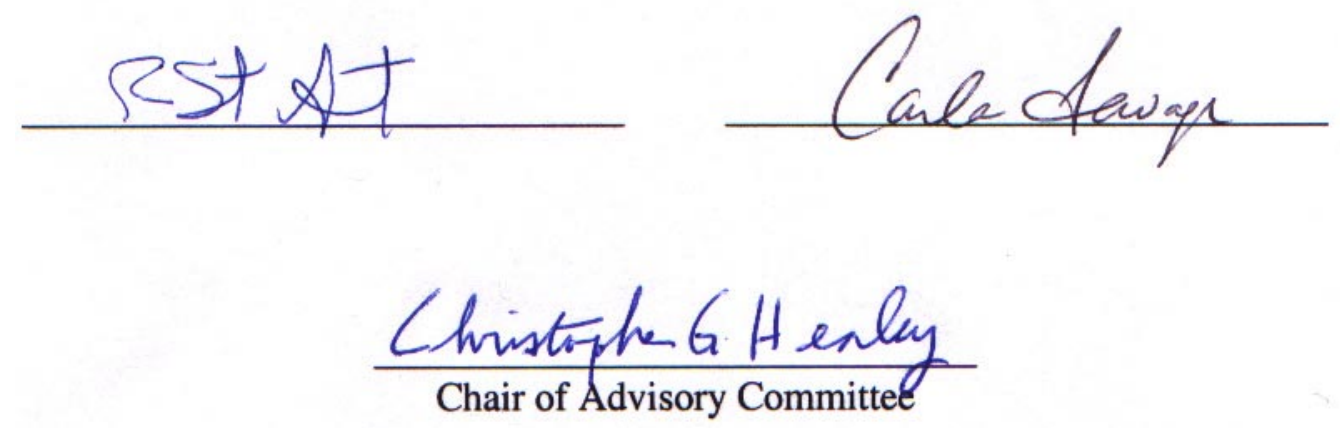


\section{BIOGRAPHY}

Brent Moorman Dennis was born July 4, 1976 in Atlanta, Georgia. In 1998, he received his Bachelor of Science degree in Mathematics with a concentration in Computer Science from Davidson College, Davidson, North Carolina. After spending a year at the University of North Carolina at Charlotte furthering his education in computer science, Brent entered the Computer Science graduate program at North Carolina State University. On October 9, 2002, he successfully completed his oral preliminaries for his Master of Science degree and will graduate in December. Brent plans to continue his academic studies with the pursuit of a doctorate degree in computer science at North Carolina State University. 


\section{ACKNOWLEDGEMENTS}

There are many people to whom I owe many thanks that have contributed to the completion of this thesis. First, I would like to thank my committee members, Dr. Christopher Healey, Dr. Carla Savage, and Dr. Robert St. Amant, for the time and effort they devoted to my thesis. A special thanks goes to Dr. Healey, my advisor and committee chair, whose critiques, high standards, advice, and support ensured I wrote a thesis to the best of my ability.

There are a number of my peers I also need to thank. A special thanks goes to Laura who shared the same thesis-writing adventure with me. She brought welcome advice and assistance throughout the creation of this thesis. Many thanks go to Sarat whose ideas and insight contributed to this thesis, even at the late hours of the day. Thanks also go to the rest

of my research group, Mike, Amit, and Vivek, who have all supported me during my time at NCSU.

Finally, I want to thank my parents and family for their unwavering encouragement and strong faith in my abilities. Their support has been instrumental to the completion of my degree. 


\section{Contents}

$\begin{array}{ll}\text { List of Figures } & \text { vi }\end{array}$

1 Introduction $\quad 1$

2 Navigation $\quad 6$

2.1 Virtual Worlds . . . . . . . . . . . . . . . . . . 7

2.2 Cognitive Maps . . . . . . . . . . . . . . . . . . . . . . 8

2.3 Supporting Cognitive Maps and Wayfinding . . . . . . . . . . . . . . . . . . 10

2.4 General Frameworks for Navigation . . . . . . . . . . . . . 13

3 Visualization $\quad 16$

3.1 Information Visualization . . . . . . . . . . . . . . . 17

3.1 .1 Overview+Detail . . . . . . . . . . . . . . 18

3.1 .2 Focus+Context . . . . . . . . . . . . . . 20

3.2 Multidimensional Visualization . . . . . . . . . . . . . . . . . 25

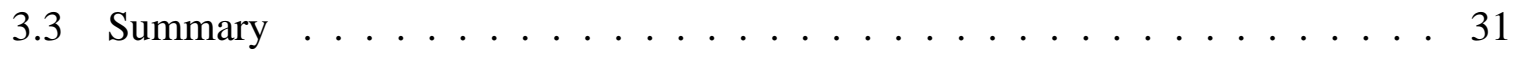

4 Areas of Interest $\quad 33$

4.1 Identifying EOIs . . . . . . . . . . . . . . . . . . . 34

4.2 Areas of Interest . . . . . . . . . . . . . . . . . . . . . . . . . . . . . . . . . .

4.3 Constructing Areas of Interest . . . . . . . . . . . . . . . 37

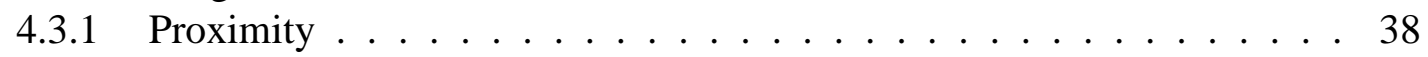

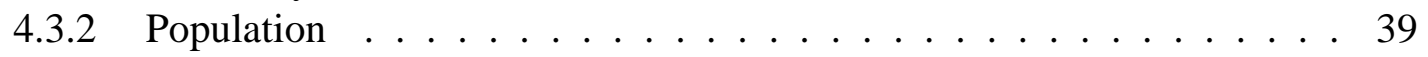

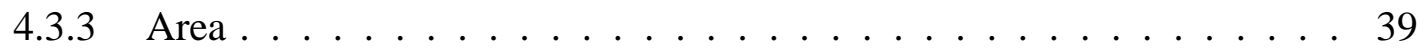

5 Graphs of the Navigation Framework 4

5.1 Discussion of Relevant Graph Theory . . . . . . . . . . . . . . . . . . . 42

5.2 Voronoi Diagrams and Delaunay Triangulations . . . . . . . . . . . . . 43

5.3 Euclidean Minimum Spanning Tree . . . . . . . . . . . . . . 45

6 Assisted Navigation $\quad 48$

6.1 Graph Traversal Algorithms . . . . . . . . . . . . . . . . . . 48

6.1 .1 Dijkstra's Algorithm ................... 49 
6.1.2 Hamiltonian Graphs and the Traveling Salesman Problem . . . . . . . 52

6.2 Optimal Viewpoints . . . . . . . . . . . . . . . . . 55

6.3 Camera Path . . . . . . . . . . . . . . . . . . . . . . . . . . . . . . 69

6.3.1 Quality Cinematic Shots . . . . . . . . . . . . . . 60

6.3.2 The Camera Path and Spline Curves . . . . . . . . . . . . . . 61

6.3.3 Maintaining Effective Exploration and Preserving Quality Shots . . . . 64

6.3 .4 User Control . . . . . . . . . . . . . . . . 65

7 Practical Applications $\quad 66$

8 Conclusions and Future Work $\quad 74$

$\begin{array}{ll}\text { Bibliography } & \mathbf{7 8}\end{array}$ 


\section{List of Figures}

2.1 Model of a navigation framework . . . . . . . . . . . . . . . . 13

2.2 Revised model of navigation framework . . . . . . . . . . . . . . . . 14

3.1 Example of a tree-map . . . . . . . . . . . . . . . . 21

3.2 Example of perspective wall and cone tree . . . . . . . . . . . 22

3.3 Example of the hyperbolic browser . . . . . . . . . . . . . . . . . . . 24

3.4 Predator-Prey Model . . . . . . . . . . . . . . . . . . . . 26

3.5 Examples of preattentive processing $\ldots \ldots \ldots \ldots \ldots \ldots$

3.6 Examples of texture elements . . . . . . . . . . . . . . . 30

4.1 Forming a cluster using convex hull algorithm . . . . . . . . . . . 38

4.2 Examples of clusters formed with varying parameters . . . . . . . . . 40

5.1 Voronoi diagram and Delaunay triangulation of a set of points . . . . . . 4 44

5.2 Example of a Euclidean minimum spanning tree . . . . . . . . . . . 46

6.1 Shortest path search using Dijkstra's algorithm _ . . . . . . . . . . . 51

6.2 Approximation of minimal cost Hamiltonian cycle . . . . . . . . . . . 54

6.3 Construction of constraint buffers . . . . . . . . . . . . . . . . 57

6.4 Optimal viewpoints and a camera path . . . . . . . . . . . . . . . 59

6.5 Natural spline curve . . . . . . . . . . . . . . . . . 63

7.1 Visualization using pexels $\ldots \ldots \ldots \ldots$

7.2 AOIs in a visualization $\ldots \ldots \ldots \ldots \ldots \ldots$

7.3 Example of an AOI tour . . . . . . . . . . . . . . . 71

7.4 Example of a shortest path tour in an AOI . . . . . . . . . . 72

7.5 Example of a shortest path from one AOI to another AOI . . . . . . . . 73 


\section{Chapter 1}

\section{Introduction}

"Science is best defined as a careful, disciplined, logical search for knowledge about any and all aspects of the universe, obtained by examination of the best available evidence and always subject to correction and improvement upon discovery of better evidence.” James Randi

With the advent of computers, scientists can now collect massive amounts of information. The volume of data currently being stored has reached a size that was unheard of only a few years ago. The analysis of large, complicated information spaces is an important challenge faced by the scientific community. Research is now focused on how to harness the power of computers to manage this data. Constructing visual representations (or visualizations) of a dataset is one technique with great potential. Effective visualizations can show both the local, detailed information represented by individual data elements and the global structure of the entire dataset. They offer an environment in which scientists can rapidly explore massive 
amounts of information. The goal of these efforts is to confirm existing hypotheses and to discover entirely new properties embedded within the data.

Visualization is relevant to many scientific areas such as medicine, engineering, earth sciences, and molecular biology [GFG $\left.{ }^{+} 94\right]$. The importance of visualization was first proposed in a 1987 NSF report [MDB87]. Panelists noted that the human brain cannot efficiently interpret large collections of data when presented in an exclusively numerical format. They suggested that visualization offers a key alternative method to examine these types of datasets. "The ability of scientists to visualize complex computations and simulations is absolutely essential to insure the integrity of analyses, to provoke insights, and to communicate those insights with others" [MDB87].

Formally, a dataset $D$ is composed of a finite set of data elements $e_{i}, D=\left\{e_{1}, \ldots, e_{n}\right\}$, where $n$ is the size of $D$. The dataset represents a set of attributes $A=\left\{A_{1}, \ldots, A_{m}\right\}$, where $m$ is the dimensionality of $D$. Every $e_{i}$ in $D$ encodes a value for each attribute, that is, $e_{i}=$ $\left\{a_{i, 1}, \ldots, a_{i, m}\right\}$.

An effective visualization should meet two fundamental criteria. First, a representation must be generated that will accurately depict the attribute values at each data element's spatial location. Second, the representation must allow a user to view the data at multiple scales, and to efficiently explore at each of these scales.

Constructing visualizations that satisfy the above criteria is a challenging problem due in large part to the size and dimensionality of a typical dataset. Traditional displays represent data using images embedded in a two-dimensional plane (e.g. a computer screen or printed page). 
To handle a dataset with $m>2$, techniques are needed to encode higher dimensional data into the plane. A second problem is visualizing large numbers of data elements. The size and resolution of a computer screen impose a hard limit on the amount of information that can be shown in a single display. As a result, some information may be forced offscreen to provide an adequate local view of the data. Successfully managing offscreen information is a fundamental problem in visualization.

Research in multidimensional visualization focuses on the first problem. These techniques attempt to display multiple attribute values simultaneously in a single image by combining multiple visual features (e.g. color and texture) to represent the attributes. However, these methods do not explicitly consider the number of data elements to visualize. If offscreen information exists, the techniques normally assume a fully-manual navigation system controlled by the user (e.g. simple translation and rotation of the view position).

Techniques from information visualization address the second problem by visualizing the dataset at multiple scales. By compressing certain parts of the display, they allow the visualization of additional data elements. Typically, there exists a user-chosen local view to provide detailed information and a global overview to provide global context about the dataset. Unfortunately, most of these techniques are not designed to visualize multidimensional data. Moreover, although they can significantly increase the number of data elements being visualized, they can still be overwhelmed by very large datasets.

Existing techniques from both multidimensional and information visualization offer only limited support for identifying and moving towards regions of interest. The ability to locate 
and display these regions of interest is critical to the exploration process. The more effort users expend searching the dataset, the less effort they may devote to analyzing it. Helping to identify the structure and location of areas of interest can greatly reduce the burden of navigating the dataset. This will dramatically increase the efficiency of the user's exploration process.

By combining techniques from multidimensional visualization and information visualization with a navigation assistant, we hope to create a system that allows researchers to easily locate and analyze areas of interest within a dataset. The navigation assistant is a software agent that aids users by identifying "interesting" data elements, structuring them into coherent regions, and reporting their locations. By revealing spatial relationships between individual elements and the regions they form, users can locate and explore within those regions more effectively. Therefore, they can focus more time on the exploration of their data and less on making correct navigation decisions.

In our system, users describe the properties of the data that classify an element as interesting. This is done with simple boolean and mathematical rules. Once elements of interest are identified, they are assigned to areas of interest using clustering techniques. Within each area of interest, a graph is built to provide local navigation assistance. The local graphs are connected to a global network to provide high-level navigation assistance between different areas of interest. Users can ask the assistant to automatically manipulate their viewpoint to aid with a variety of navigation tasks, such as moving to a nearby area of interest and viewing the data elements it contains. Automated tours are built using the graph framework, graph traversal algorithms, optimal viewpoint construction, and camera planning techniques. At all times, 
users maintain full control of the camera, choosing to halt (or initiate) automated tours or to manually navigate to any location in the data.

The remainder of this thesis is structured as follows. Chapter 2 discusses research conducted in the area of navigation. Chapter 3 describes systems in both multidimensional and information visualization. Chapter 4 explains how areas of interests are formed. Chapter 5 details the construction of the graph components of the framework. Chapter 6 defines how the framework is used for assisted navigation. Chapter 7 describes a practical application of the framework to a real world dataset. Finally, Chapter 8 contains future work and conclusions. 


\section{Chapter 2}

\section{Navigation}

With the advances in computer graphics and computer processing power, scientists and engineers are now able to create large virtual worlds. As virtual worlds increase in both size and application scope, users must contend with the problem of visualizing and navigating within those virtual spaces. "Becoming lost" in virtual worlds has been identified as a serious hindrance to users [DMR90]. Visualizations are a type of virtual world. They emulate the physical world by converting a dataset into a virtual environment of objects (data elements) each assigned to a relative position. Users can change their location within the world and interact with its objects. Therefore, reviewing research in navigating virtual worlds will be useful for understanding how to effectively navigate a visualization.

Navigation within a virtual world or information space is a growing area of research in information and computer science. Navigation is a basic human process that we perform every day. Humans use a wide variety of tools along with cognitive structures and activities to 
navigate the physical world. Researchers have discovered that effective techniques for navigating the physical world may not always apply to a virtual world. This consequence has led to investigations about how humans perform successful navigation tasks. Discovering the underlying foundations of human navigation might provide researchers with information on how to successfully support navigation within a virtual world.

\subsection{Virtual Worlds}

Virtual worlds can be subdivided into two main categories: discrete and continuous [Mod97]. Discrete environments are typically document-based hypermedia, such as the World Wide Web or a document database. Continuous environments often use a spatial model that simulates the physical world. Virtual reality systems or a satellite image display are examples of a continuous information space. Discrete spaces "take advantage of well-developed human skills in organizing and classifying information," while continuous spaces use "well-developed human cognitive and neuro-motor skills for dealing with the physical world" [Mod97].

Darken further classifies virtual worlds using three attributes: size, density, and activity [DS93]. He describes a world as small if it can be mostly viewed from a single viewpoint such that all pertinent and important distinctions between objects are discernible. As motivation for his definition, Darken uses Kuipers and Levitt's definition of a large world, "a space whose structure is at a significantly larger scale than the observations available at an instant"[KL88]. If no vantage point exists from which the entire world can be viewed in detail, Darken classifies the world as large. The density of a world refers to the amount of empty space between environ- 
mental objects. Sparse worlds have large amounts of open space between objects, while dense worlds have relatively large numbers of objects and much less open space within the environment. Activity refers to whether or not objects change within the environment. Static worlds contain objects that do not change in appearance or position over time. Dynamic worlds can be much more complex since objects have the potential to change in appearance and position.

\subsection{Cognitive Maps}

Psychologists are very interested in discovering and understanding the cognitive structures humans use for navigation. Tolman first proposed the concept of cognitive maps for navigation tasks [Tol48]. Cognitive maps, or mental maps, are a crucial tool used by humans for navigation. During navigation tasks, cognitive maps are critical for storing and using environmental information. Tolman built his theory on the results of experiments conducted with rats in mazes. He discovered that the rats acquired a "field map of the environment" based on knowledge gained during the process of learning [Tol48]. He further described this knowledge as "a cognitive-like map of the environment ... indicating routes and paths and environmental relationships" [Tol48].

Experts have been unable to determine if cognitive maps are analog (continuous) or propositional (discrete). Stevens and Coupe conducted a set of directional experiments whose results support human storage of environmental knowledge in a discrete hierarchical representation [SP73]. Their research found that their subjects' directional judgments of geographical locations can be significantly distorted by large, surrounding areas. This implied humans do not 
accurately store navigation information in an analog fashion. The authors state it is not efficient for humans to store every possible spatial relationship in their long-term memory, making hierarchical representations ideal for both the storage and retrieval processes [SP73].

Downs and Stea proposed an entirely different model. They suggest cognitive maps are stored in an analog representation [DS73]. Downs and Stea believe that humans use locational information, composed of distance and direction, and attributive information, composed of description and evaluation, to build a diversified group of "signatures" [DS73]. Signatures are sets of coding and decoding operations performed by humans. Signatures resemble cartographic maps, linguistic information, and other kinds of visual imagery. Noting human limitations on information storage, Downs and Stea acknowledged that "cognitive maps are complex, highly selective, abstract, generalized representations in various forms ... incomplete, distorted, schematized, and augmented, and we [found] that both group similarities and individual differences exist" [DS73].

Thorndyke and Hayes-Roth also support an analog representation of mental maps [THR82]. They proposed that humans acquire special types of spatial knowledge from maps and the navigation process. Route (or procedural) knowledge stores procedures used to move through an environment, while survey knowledge stores properties of an environment's topography. As navigators gain more experience in an environment, they tend to transform route knowledge into survey knowledge. Research shows different individuals favor using either route or survey knowledge [Pas84]. Another type of spatial knowledge was presented by McKnight [MDR93], who identified the storage of basic feature recognition as landmark knowledge. These three 
types of spatial knowledge are key resources for successful navigation.

\subsection{Supporting Cognitive Maps and Wayfinding}

Within an environment, certain objects or combinations of objects possess properties that can have a large influence on a mental map. Lynch proposed a set of structural design principles that can improve the function of mental maps by increasing the imageability of the environment [Lyn60]. Lynch defines imageability as

“...that quality in a physical object which gives it a high probability of evoking a strong image in any given observer. It is that shape, color, or arrangement which facilitates the making of vividly identified, powerfully structured, highly useful mental images of the environment" [Lyn60].

Lynch defined five structural components that improve the imageability of an environment.

1. Edges: linear borders between two areas.

2. Paths: conduits of movement, considered to be the most essential of the five elements.

3. Districts: two-dimensional regions of an environment which have distinguishing characteristics from each other. Observers may mentally enter and leave a district.

4. Nodes: similar to districts, except they are much smaller and occur in vital, important positions. Typically, nodes serve as hubs and gathering places.

5. Landmarks: well recognized objects. 
Well-designed environments should integrate these components into a concrete underlying structure to afford the creation of better mental maps. While Lynch's work dealt with city planning, his concepts have strong potential for application to virtual worlds. The use of Lynch's ideas in virtual worlds has been studied in [CIP96, DS96, IB95, Mod97, Shu90].

Passini defined wayfinding by expanding on Lynch's ideas. He claims wayfinding is composed of three iterative processes [Pas84]:

1. Build a cognitive map and collect information (temporal, locational, and descriptive) to better understand the environment.

2. Build a plan of actions to take within the environment based on the information that has been gathered.

3. Perform the planned actions within the environment.

Passini proposed a set of design components derived by the revision of Lynch's imageability design elements. Passini's three design elements that support effective wayfinding are:

1. Create an organizational principle that promotes imageability and supports efficient cognitive processing.

2. Create a spatial enclosure which supports both memorability as well as contextual and structural inferences.

3. Create a spatial correspondence between important environmental properties and components to facilitate the development of cognitive maps 
Darken and Sibert explored how humans use the wayfinding process. They classified wayfinding tasks into three different categories [DS96]:

1. Naive search: a searching task in which the navigator has no presumptive information on the target's location.

2. Primed search: a non-exhaustive searching task in which the navigator knows the target's location.

3. Exploration: any type of wayfinding task in which there is no pre-defined target.

These classes are mutually exclusive, but practical wayfinding tasks are typically sequences of the above searches. For example, a navigator could have a general idea of where a target might be located, but its specific location is unknown. Therefore, a navigator may take a primed search to the general area of the target, and then perform a naive search of the target's neighborhood [DS96]. Naive searches can be much more common in virtual worlds than they are in the physical world, since first-time explorers of a virtual world may lack adequate route and survey knowledge of the environment.

The authors say that an environment must support both exhaustive (naive) and non-exhaustive (primed) searches. Most importantly, wayfinding should support the acquisition of survey knowledge. While naive and primed searches attempt to locate a specific target, the goal of exploration is to gather survey knowledge of the environment. Therefore, a good wayfinding design should also facilitate the development of survey knowledge independent of the wayfinding task performed by the navigator. Darken and Sibert defined three environment organizational 


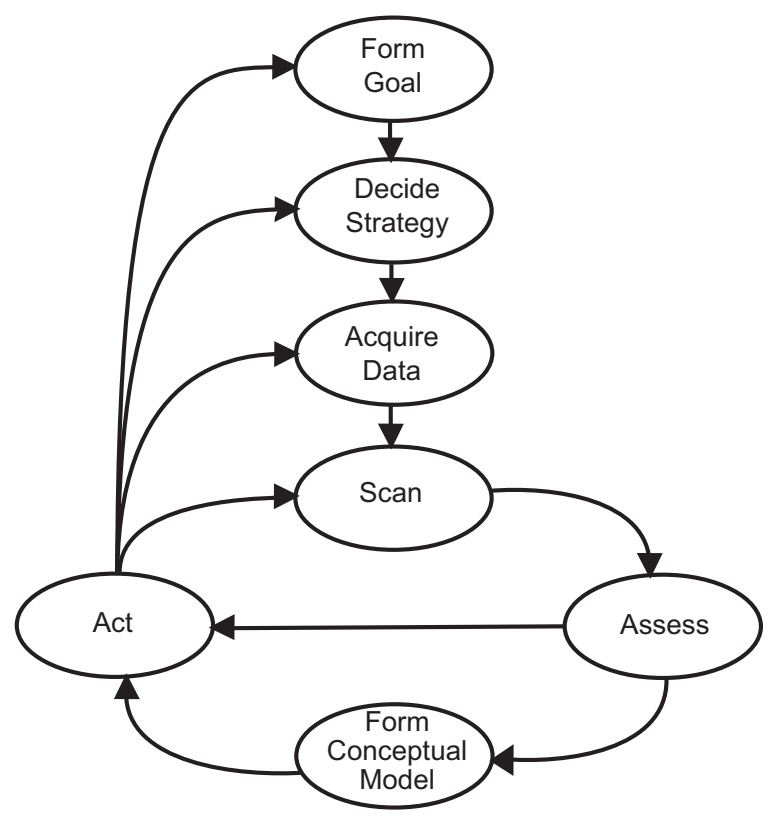

Figure 2.1: A general navigation framework model.

principles for the support of wayfinding that follow Passini's elements [DS96]:

1. Divide the world into distinctive regions while preserving a sense of place.

2. Organize the regions under a simple organizational principle.

3. Provide frequent directional cues.

\subsection{General Frameworks for Navigation}

Darken defined navigation as the composition of wayfinding and locomotion [JF97]. Environmental features that support an effective framework for wayfinding in a virtual world have been described above. General framework models for navigation are often composed of wayfinding cognitive activities and locomotion procedures based on the results of those activities. 


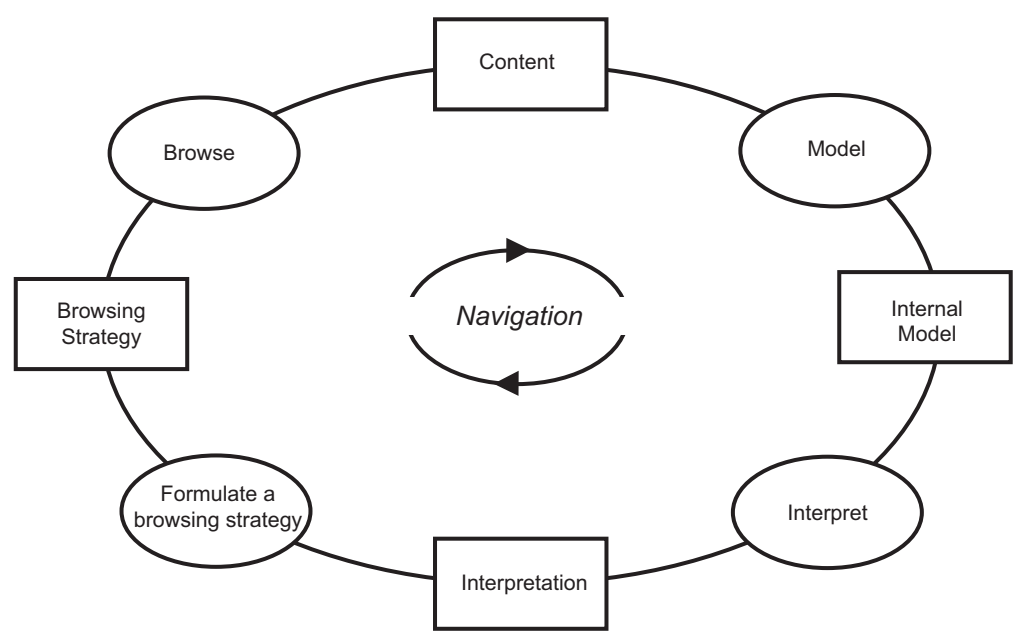

Figure 2.2: Spence's revised navigation framework model.

At the same workshop, Spence proposed his general framework for navigation (Figure 2.1) [JF97]. With this framework, the navigator's goal is influential to the development of the appropriate navigation strategy. The navigator gathers a collection of data to help perform the appropriate navigation task, for example, reading a map, querying a search engine, or accessing some other external source. The navigator then scans the environment and makes an assessment as to whether his expectations are met and if he is closer to achieving his goal. This assessment contributes to the modification of the navigator's cognitive map of the environment. The assessment also influences the action, locomotive or cognitive, taken by the navigator. Possible actions are very diverse. The action might be either locomotive, e.g. backtracking or turning right, or cognitive, e.g. modifying the navigation strategy or collecting more environmental information. The navigator could also abandon the current goal and begin the construction of a new goal.

Spence later revised his general navigation framework (Figure 2.2) [Spe99]. His new model 
is composed of four cognitive activities: browsing, modeling, interpreting, and formulating the browsing strategy. When performed in sequence, these activities constitute the process of navigation. The result of each activity influences the execution of the next. Spence describes the browsing process as the "registration of content" [Spe99]. Information is gathered but not yet integrated into any type of internal environmental representation. The subsequent stage performs the integration of information into the users' cognitive maps. The cognitive map is the users' mental model of the environment. Users rely on their model and the display of the data to develop an interpretation of the environment. Interpretations influence the formation of subsequent browsing strategies. For example, if the interpretation is that the user is in the neighborhood of a target, the strategy might change from a general scan of the data to a detailed inspection of a small region in the data. The cognitive map also influences the building and execution of a browsing strategy. Spence points out that existing internal models can influence the construction of the cognitive map of the navigation space and the development of browsing strategies. 


\section{Chapter 3}

\section{Visualization}

The advancement of graphics technology has enabled the presentation of scientific data to evolve from simple graphs, scatter-plots, and tabular forms to more complex graphical representations. These representations can allow scientists to more easily interpret information and relationships from their data than if they were only studying a table of empirical numbers. Likewise, the increase of the amount of data that can be stored electronically has allowed the creation of huge databases and virtual libraries that can be accessed from a single graphical display. Electronic storage of information is more space and time efficient than conventional paper mediums making it ideal to archive today's vast quantities of data. However, locating and accessing this information is not a trivial task. Users need navigation tools to locate information and translation tools to convert the data from 1's and 0's to a format users can efficiently interpret. Visualization attempts to find solutions to these problems.

As defined by Card, Mackinlay, and Shneiderman, visualization is "the use of computer- 
supported, interactive, visual representations of data to amplify cognition" [CMS99]. Cognition can be defined as the acquisition and use of knowledge. Expanding cognition increases the ability of users to perform discovery, decision-making, and explanation. These cognitive activities can provide users with extended insight into a dataset.

The concept of visualization formally began with an NSF report in 1987. This report envisioned visualization as a tool that allowed the handling of large sets of data in order to aid scientists with gaining insight and observing underlying trends and relationships within the datasets [MDB87]. Visualization techniques are sometimes divided into two different categories: scientific visualization and information visualization. Scientific visualizations are built to display physically based data that possesses some form of inherent geometry. Information visualization attempts to display non-physically based, or abstract, data forms.

Below is a discussion of several visualization systems that have been built to address scientific or abstract data. Given the previous discussion of how the dimensionality of modern datasets continues to grow, we will focus on multidimensional scientific visualization techniques.

\subsection{Information Visualization}

Some information visualization systems attempt to fit large amounts of information on a single screen. One group of techniques designed around this goal are the focus + context and overview + detail algorithms. overview + detail presents users with a global overview of a dataset, while providing interactive mechanisms for accessing local, detailed information. focus + context 
presents users with global contextual information along with ways to focus on local areas to collect more detailed information. Many information visualization systems may fit into either of these broad categories.

\subsubsection{Overview+Detail}

While not a requirement, overview + detail algorithms typically provide a user with multiple, separate views of the data. Each view presents the dataset to the user at a different scale or view. One of the critical scales is the overview. An overview "reduces search, allows the detection of overall patterns, and aids the user in choosing the next move" [CMS99]. However, users also need to be able to rapidly access detailed information about local areas of the dataset. Therefore, a detail view should also be provided. Multiple views can be presented in two ways: one at a time (time multiplexing) or simultaneously in different sections of the screen (space multiplexing) [CMS99]. Multiple views occupying the same screen requires additional screen area, forcing space-multiplexing algorithms to make calculated trade-offs with the use of available display space. A variety of overview + detail systems have been built [Shn92, KPS97, BH94, $\mathrm{PMR}^{+}$96, MF95].

Plaisant et al used overview + detail techniques to construct a tool to visualize medical and court documents called LifeLines [PMR $\left.{ }^{+} 96\right]$. Their system presents a medical or court professional with an overview screen of a personal record. For the period of time a record represents, the system uses color-coded facets to symbolize time periods in the subject's life (e.g. open criminal cases or medical conditions). Specific icons are used for important events such as 
arrests or doctor's appointments. The information is presented below a time-line which marks units of time (e.g. days, months, or years) with vertical bars. Dividing the display area into time blocks supplies the user with a sense of the scale within the overview and allows the user to better understand the context of events. For large records occurring over lengthy time periods, LifeLines condenses the facets and reduces the width of the blocks of time. Also LifeLines drops off less important information, such as labels and tag-names for the facets and icons. Since low-level detail can be lost with large files, the authors propose two methods for retrieving it. If enough screen space is available, LifeLines will use multiple screens to display an overview and a number of detailed views. If only one view is allowed, LifeLines enables users to zoom in to areas by clicking on a location of the display, thus revealing facet thickness, labels, and other details. This process is known as semantic zooming and was first presented by Bederson [BH94].

Semantic zooming occurs when the content remains constant while the representation of that content changes. Bederson proposed that at zoomed-in scales, details of an object should be available to the user. However, zoomed-out views might be more effective if an object takes on a representation different than the traditional scaled-down view [BH94]. Bederson implements this concept with $\mathrm{Pad}++$ [BH94, Bed95]. Pad++ is a zooming interface that supports colored text, text files, hypertext, graphics, and images. Users are able to define distinct representations for the above objects at different scales. Pad++ was used to visualize a file system. At the top level, the user is presented with square frames for directories. As zooming occurs, individual files are represented by colored squares. As the user continues to zoom, the square is 
replaced by a representation of the contents of the file, for example the hypertext of an $H T M L$ file. Furnas used space-scale diagrams to help build different representations of the same object at different scales for semantic zooming [FB95].

The tree-map is another well-known overview+detail system (Figure 3.1)[Shn92]. The tree-map decomposes a dataset into a rectangular image. This image contains individual regions which are hierarchically partitioned, usually into rectangles, based on the different attributes of the data. The tree-map can display high levels of detail by zooming into regions in which the user is interested. By clicking on the display, the appropriate region is expanded, replacing the overview display and revealing more high-level details. Shneiderman also uses the tree-map to view the contents of a file system. The application of tree-maps has been studied in other areas, including software engineering and tournament monitoring [JB97, BE94].

\subsubsection{Focus+Context}

Another technique that researchers use in information visualization is focus+context. Like overview +detail, focus+context provides users with both global overview information (context) and local detail (focus) information. However, focus + context techniques often try to present both types of information in a single display. This design aspect is based on an assumption that the user requires both overview and detail information simultaneously. However, information needed from an overview might be different than information needed from a focus view. This condition allows focus + context techniques to selectively reduce information from a display by filtering, warping, or scaling local detail in an attempt to allocate more display 


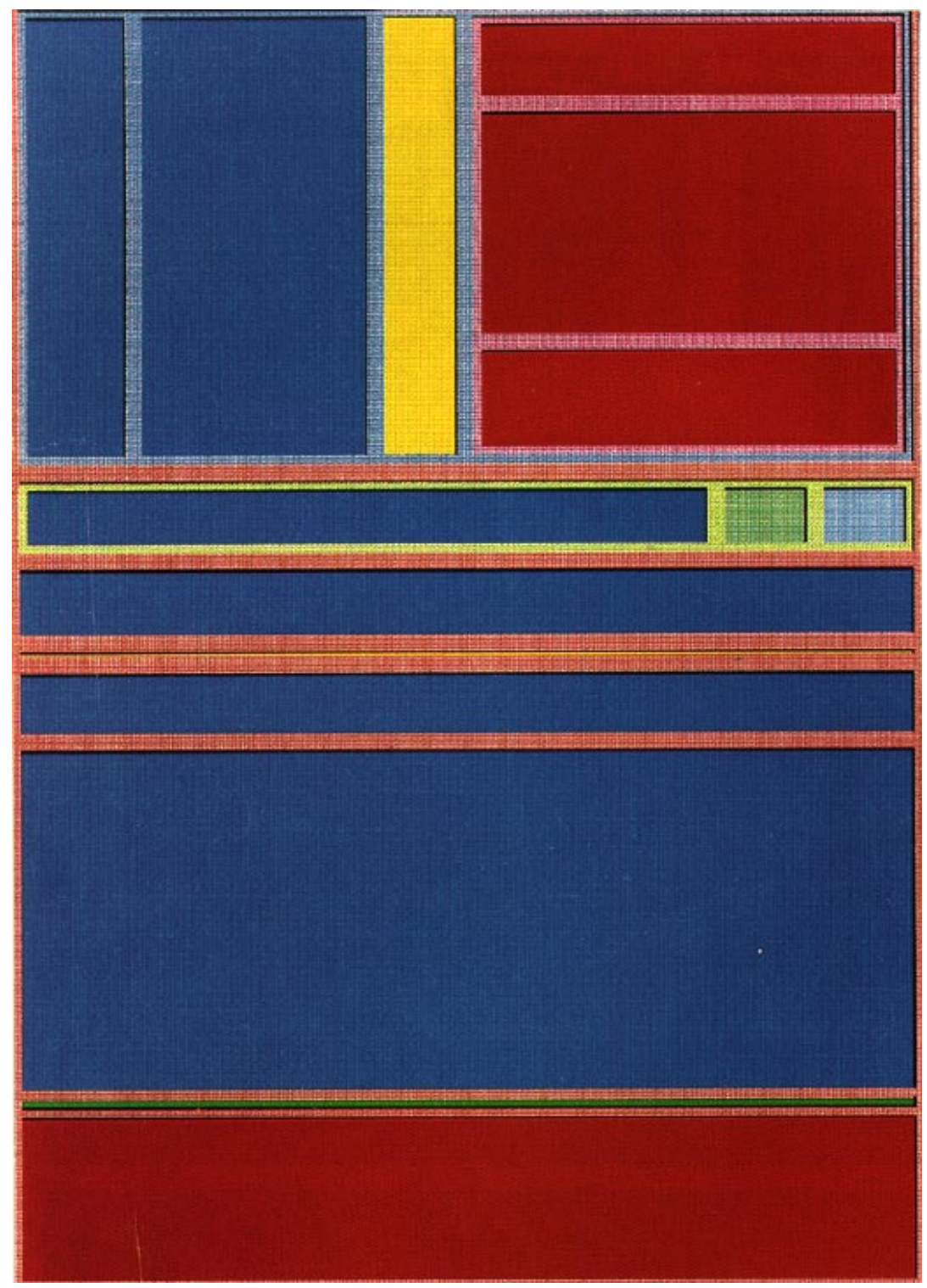

Figure 3.1: An example of a tree-map visualizing a filesystem with 15 files at 3 levels. The map is initially segmented into 8 blocks. The top two blocks of the first level are divided again, producing a new level. The dark red block located in the upper-right corner of the map is partitioned again to form the third level. 


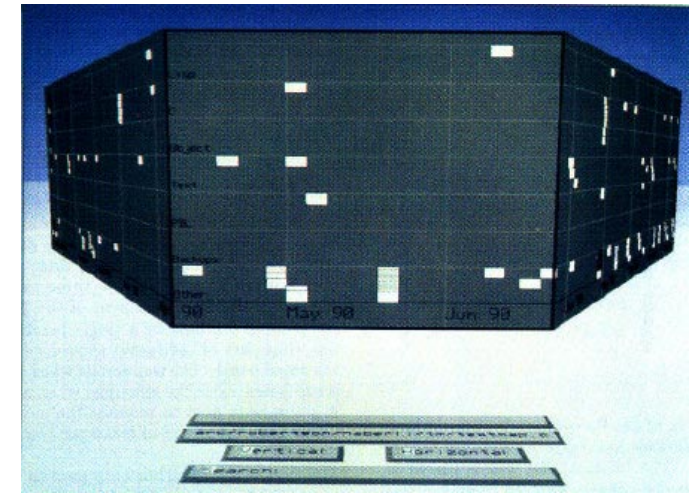

(a)

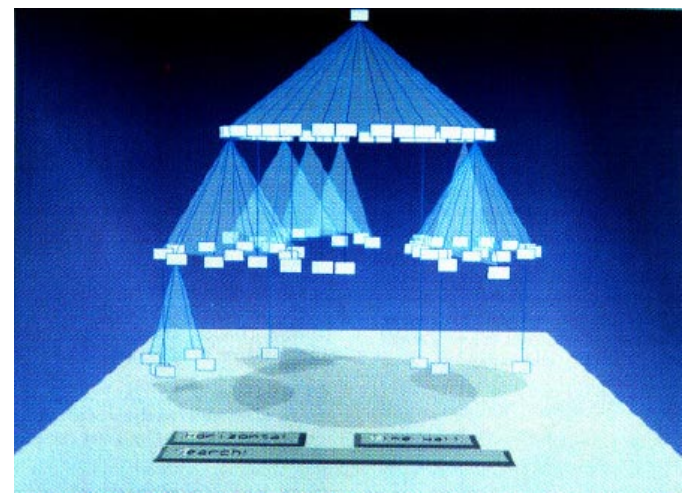

(b)

Figure 3.2: (a) shows a perspective wall with a central focus view and two overviews that are warped to smoothly unify the three different displays. (b) shows a cone tree with shadows added to assist a viewer in understanding the structure of the cone tree.

resources to correspond to the interest of the user's attention [CMS99].

The bifocal display is an early focus +context technique that explicitly provides the user with a focus and contextual display [SA82]. This system presented the user with a single focus view flanked by two overview displays. However, this system did not seamlessly unify the contextual views into the focus display, thus providing no assistance for integrating the different scales of information. The perspective wall was designed to smoothly unify the focus view with contextual views (Figure 3.2a) [MRC91]. By warping the overview displays, this system assists users with the integration of focus and contextual information.

The cone tree uses depth and perspective projection to visualize hierarchical data (Figure 3.2b) [RMC91]. Cone trees lay out hierarchical data in three dimensional space. A level's root is placed at the apex of a cone while the nodes are evenly distributed along the cone's base. The cones' heights are uniform from level to level, while the radius of a cone's base will diminish as one travels down the hierarchy. Also, the body of a cone is transparent, allowing 
users to visualize information that would otherwise be occluded from their view. The cone tree supports interactive controls that allow a user to bring components into or out of focus. Specifically, the user can perform pruning or growing operations that increase or reduce the amount of information in the display.

Cone trees harnessed properties of perspective projection in Euclidean geometry. Instead of Euclidean geometry, the hyperbolic tree uses properties of hyperbolic geometry to visualize additional information. In Euclidean geometry, for a given point $p$ and line $l$, there exists only one line passing through $p$ that is parallel to $l$. In hyperbolic space, there exists multiple parallel lines that diverge from each other. When a hyperbolic plane is placed within a Euclidean two dimensional circular disc, the size of components will contract as the distance from the center of the circle increases. Also, there is an exponential growth in the number of available display regions, although with a corresponding reduction in each region's size. If used to visualize a tree, the hyperbolic browser can fit a large number of nodes near the circumference of the disc. As nodes are moved to the center of the disc, more display space becomes available allowing the presentation of detailed information for a node.

The hyperbolic browser uses properties of hyperbolic space to visualize a dataset in a "fisheye" display. A fisheye view provides local detail at the center of the view. As one moves outwards, the view will fade to mostly contextual information. Often, the local detail fades rapidly as one moves away from the center. As a result, a fisheye succeeds as a powerful focus + context tool that can provide both contextual and detailed information of a dataset in a single view. Furnas first published a report on the use of fisheyes for information visualiza- 


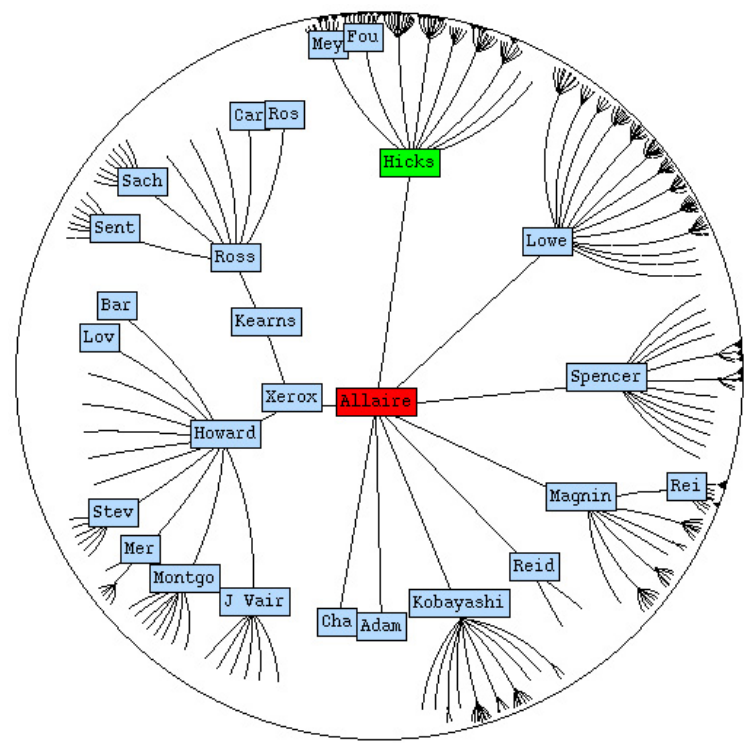

(a)

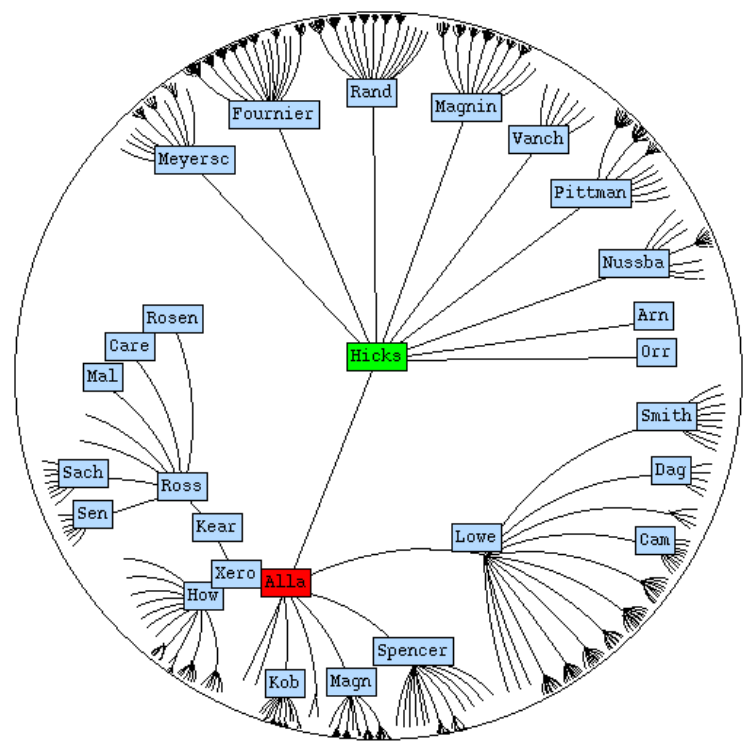

(b)

Figure 3.3: A hyperbolic browser is shown in (a). (b) shows how a node is moved out of focus and new node is brought in to focus.

tion [Fur81, Fur86]. He proposed the use of a Degree of Interest, or DOI, metric to determine the amount of detailed information to display for a given component. The DOI metric is a function of a distance and a Level of Detail, $L O D$, function. Distance is a value representing a system dependent length between a given component and a focus point. For every component, the $L O D$ provides a measure of that component's importance, particularly to the global structure. Customized fisheye views can be built for a dataset and a user's goals by defining the appropriate $D O I$, distance, and $L O D$ functions. Fisheye views have been used in a variety of document viewers [BHDH95, RM92, RC94, SSTR93]. Sarkar and Brown extended Furnas' general fisheye concepts to include consideration of the layout of the content of a dataset [SB92]. Further work for visualizing graphs and layout techniques for graphs can be found in [Noi93, SZG ${ }^{+}$96, SB92, BETT99]. 


\subsection{Multidimensional Visualization}

Information visualization techniques try to resolve the complications of visualizing a large number of data elements. What happens when each data element represents more than one data attribute? Refining a multidimensional dataset into individual displays representing individual attribute values can be counterproductive to understanding the data. The goal of multidimensional visualization is to find an effective visual summary of datasets with $m>1$ so that users can find important relationships among the attributes. [WB97].

A simple example is the well-known biology model of the predator-prey relationship. A biologist builds a mathematical model and uses it to generate $D$. For this particular $D, A$ is composed of three attributes, time, prey population, and predator population. The biologist constructs two graphs, one representing time versus predator population and the other time versus prey population (Figure 3.4). Both graphs are placed in the same display, one on top of the other. Immediately, the biologist can observe how his model suggests the strong interdependency between predator and prey populations.

Building and comparing scatter plots and curves is one of the earliest forms of visualizing multidimensional data. However, as the number of attributes grows, such composite images can become too congested. This can prevent researchers from detecting features and relationships within their data. The HyperSlice and hyperbox techniques attempt to relieve this congestion by segregating the data into plots comparing two attributes [vWvL93, AC91]. Instead of a single, unified image, there now exist a set of images, each showing the relationship of two attributes. These techniques depend on the user's ability to selectively focus on regions and the 


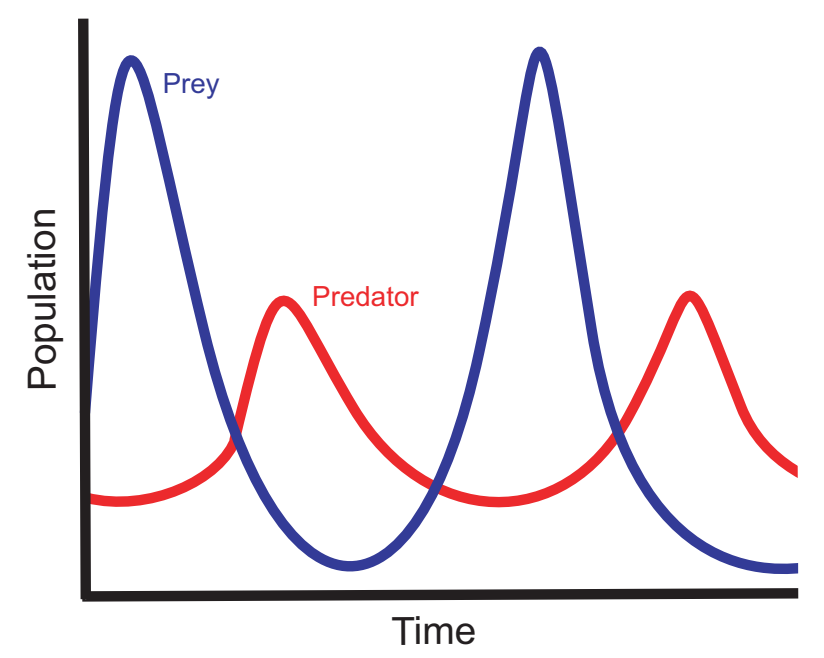

Figure 3.4: A two-dimensional graph of the relationship between predator and prey populations.

system's ability to create effective layouts for successfully visualizing multidimensional data. However, the disjoint nature of these visualizations can impede a user in detecting relationships involving more than two attributes.

Color is a powerful tool for visualizing information. Ware studied some of the strengths and limitations of visualizing data with color [War88]. He investigated how background color can interfere with the perception of a foreground color. His experiments tried to determine if users can accurately read information from univariate displays despite the effects of this contrast interference. Ware points out that while users could perform accurate scans, the construction of an appropriate colormap is often dependent on the users' tasks. Bergman et al attempted to build appropriate colormaps based on properties of the dataset and human perception [BRT95]. They constructed a rule-set that selects colormaps based on data type and spatial frequency, as well as the user's task (generalized to segmenting or highlighting components of the data). 
These rules derived values for the luminance, hue, and saturation of a colormap.

Color is a preattentive visual feature. Preattentive visual features can be detected by the low-level human visual system with great accuracy and high speed, typically in under 200 milliseconds. Moreover, preattentive processing operates independent from the number of elements in a view. Examples of preattentive tasks include edge detection, target identification, and region tracking. In data exploration, preattentive processing is an extremely powerful tool for visualization. Well-designed visualizations can combine preattentive visual features in ways that allow users to rapidly detect important characteristics of the data.

However, visualizations must map preattentive visual features to attributes with care. When used in conjunction, certain combinations of visual features can interfere with preattentive processing. Consider the visual features of color and shape (see Figure 3.5). A red circle can quickly be identified among a group of blue circles. Likewise, a red circle can be preattentively located among a group of red squares. Unfortunately, among a group of red squares and blue circles, a red circle is difficult to locate. The low-level vision system can not preattentively integrate the color and shape visual features.

In order to harness the power of preattentive processing, research has steered towards developing visualizations using texture. Some of the early research on how the human visual system processes texture information was conducted by Julész [Jul81]. Julész proposed a texton theory that claims the low-level visual system detects three types of feature: elongated blobs with a given set of visual properties (e.g. orientation and length), ends of line segments, and crossings of lines. Julész's research demonstrated that the low level visual system cannot 


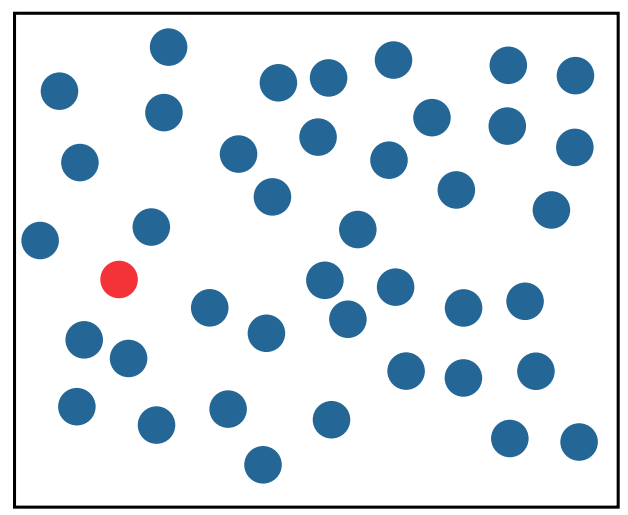

(a)

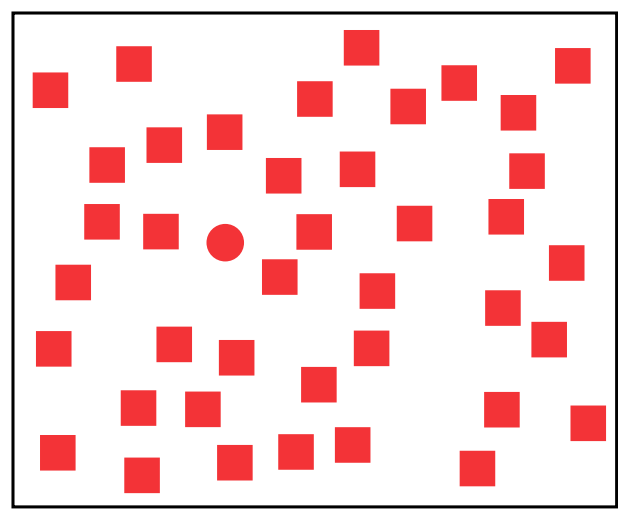

(c)

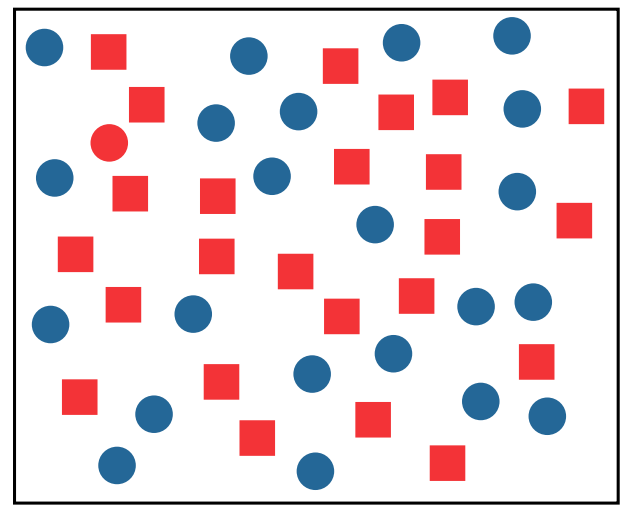

(e)

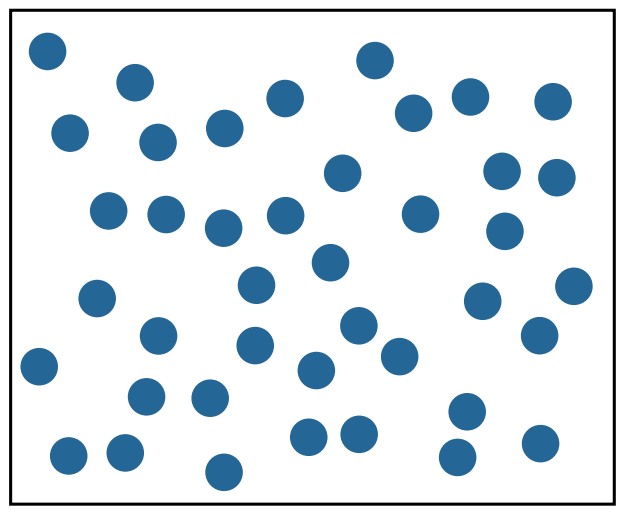

(b)

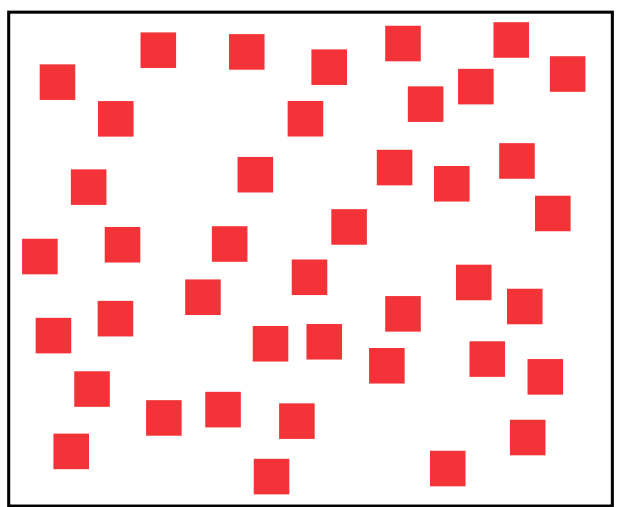

(d)

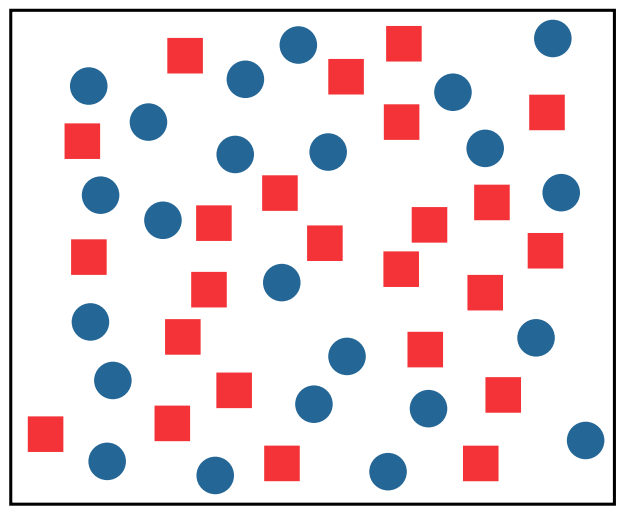

(f)

Figure 3.5: Examples of target searches: $(a, b)$ demonstrates how the search for a red circle in a sea of blue circles is rapid and accurate, target is in (a). (c, d) demonstrates how the search for a red circle among a sea of red squares is rapid and accurate, target is in (c). (e, f) demonstrates how identifying another red circle among a sea of red squares and blue circles is significantly more difficult, target is present in (e), and absent in (f). 
distinguish between textons that belong to the same category.

Grinstein and Pickett implemented a system, EXVIS, that demonstrates Julész's theories [PG88, GPW89]. EXVIS constructed "stick-men" to visualize spatial coherence within a dataset (Figure 3.6a). One stick-man is associated with each data element. Attribute values in the element orient the limbs of its stick-man [PG88]. When they are displayed, the stickmen form texture patterns that identify spatial regions with common sets of attribute values. Boundaries between such regions are also easily identifiable. The original stick-man icon used a five limbed figure to represent a dataset with five dimensions. Four dimensions modified the orientation of the four limbs of a stick-man, while the fifth dimension determined the orientation of its body. EXVIS belongs to a family of visualization systems called iconographic systems. Iconographic systems use glyphs or icons to integrate texture properties into a visualization. The geometric appearances of the icons are determined by the attributes of a dataset.

Ware and Knight constructed their own textons, based on Gabor filters, to create texture visualizations [WK95]. Ware and Knight built the OSC model of texture space with three scaling values: orientation $(\mathrm{O})$, size $(\mathrm{S})$, and contrast $(\mathrm{C})$. The authors note that this model is not necessarily complete. Orientation refers to how the textons were oriented within the environment. Size refers to the physical size of the textons. Contrast refers to the ability to distinguish a Gabor element from its background. Gabor filters were applied to Gabor elements in order to vary the OSC scales of the elements. Ware and Knight incorporated a three dimensional color model into their visualization techniques, allowing them to represent five dimensions of data. Three dimensions were represented by the OSC components and the 


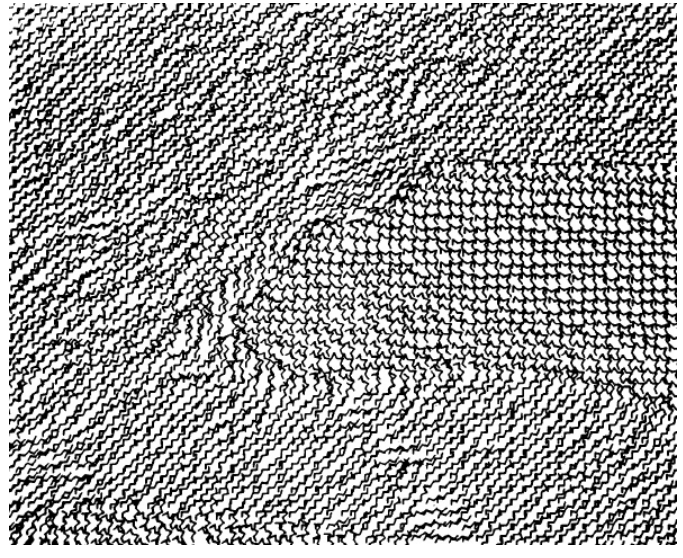

(a)

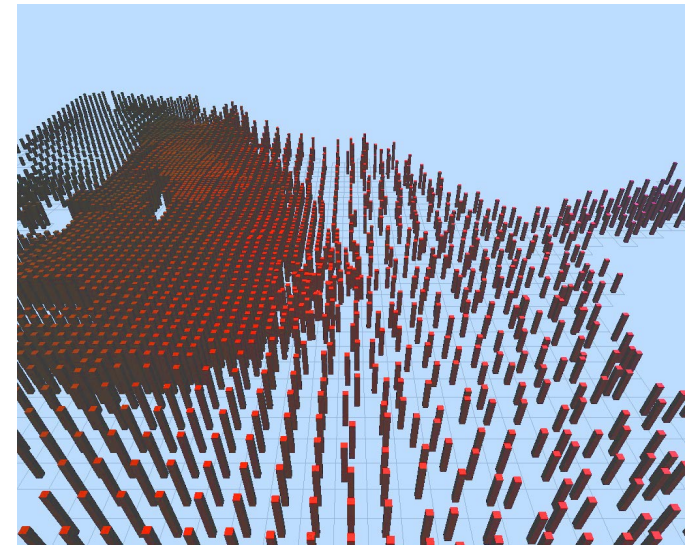

(b)

Figure 3.6: (a) shows a visualization using EXVIS stick-men and (b) shows how pexels are used to visualize a weather dataset.

remaining two were represented by two color components. The third color component was needed to properly visualize texture by providing an appropriate background color.

Healey and Enns sought additional ways to combine color and texture features in a multidimensional visualization. They constructed perceptual texture elements, or pexels, to represent multiple attributes with color and texture properties at a single spatial location (Figure 3.6b) [HE98, HE99]. Healey and Enns specifically studied the texture features of height, density, and regularity. They conducted experiments to determine how texture features can interfere with or facilitate preattentive processing. These experiments revealed that users could identify fluctuations of height with great ease. It was slightly more difficult for users to differentiate between density variations, while users had a significantly more difficult time distinguishing variations in regularity. As a result, Healey and Enns ranked the texture features to provide a guide for creating effective visualizations that use these texture properties. The most important attribute should be ranked to height, the second to density, and regularity mapped to the least impor- 
tant attribute. Healey and Enns also studied the influence of color on preattentive processing. They found that colormaps composed of three to five colors could be discerned preattentively, while larger colormaps could not. Other experiments were conducted to determine how color and texture features interact with one another. These experiments revealed that color variation caused a small, yet significant, interference with the detection of regions with certain height and density characteristics. However, texture features did not interfere with the detection of regions defined by color.

\subsection{Summary}

Multidimensional visualization techniques use layout and visual features (e.g. color and texture) to display multidimensional datasets in a two dimensional planar image. These multidimensional techniques focus mainly on handling large $m$ values. None attempt to visualize efficiently a large number of data elements. It is left to users to locate offscreen data elements using simple navigation tools, such as translation and rotation of the viewpoint. Information visualization techniques provide local and global information simultaneously to users to display large numbers of data elements effectively. However, these systems are not concerned with effectively visualizing data elements composed of multiple attributes. Information visualization techniques often filter local information to provide global context views. How should these filters be modified to provide the necessary overview of a multidimensional dataset? Should certain attributes be given precedence over others during the filtering process? The system in this thesis combines techniques from information and multidimensional visualization to 
display large numbers of multidimensional data elements. Furthermore, this system operates under the assumption that information will be omitted from the current view and attempts to provide navigation support for locating these offscreen regions of interest. 


\section{Chapter 4}

\section{Areas of Interest}

Every dataset contains elements that a user considers interesting. These elements encode in-

formation that defines the underlying properties of the dataset. A key part of data exploration is locating these elements and enabling the user to examine them. To increase the efficiency of an exploration task, a visualization system can highlight these interesting data elements and inform the user of their positions. Reducing the users' search efforts increases the likelihood they will gain additional insight into a dataset. Our system provides the above functionality and takes it one step further. By organizing elements of interest into larger entities, our system provides additional support for studying interesting properties of a dataset and reducing the burden of navigation. 


\subsection{Identifying EOIs}

Identifying elements of interest, or EOIs, is an important step in the navigation process. Identification should be simple, accurate, flexible, and rapid. Once identified, EOIs can be incorporated into the navigation framework. The framework will serve as a backbone for providing users with tools to more easily explore the EOIs in a dataset.

Most data elements can be classified as "interesting" based on properties of their attribute values. Consider an e-commerce auction dataset where the data elements are composed of the attributes bidderID, itemID, currentbid, and time. Users might need to examine data elements whose current bids are much higher than average in order to view who is winning certain auctions. Similarly, users might wish to explore data elements at the end of the auction, or data elements where the time attribute is highest, in order to observe any rapid increase in bids that may occur as an auction closes.

All of these properties can be explicitly defined using a simple mathematical grammar. Using mathematical and boolean operators with a dataset's attributes as operands, a set of rules can be generated by the user that will classify an element as interesting. As an example, consider the following potential set of rules for a collection of weather data.

$$
\begin{aligned}
\text { temperature } & >\operatorname{avg}(\text { temperature })+2 * \text { stddev(temperature }) \\
\text { windspeed } & >\text { median }(\text { windspeed })+10.0 \\
\text { precipitation } & \leq 3.0
\end{aligned}
$$


These rules identify data elements that represent locations characterized by higher than normal temperatures (Equation 4.2), wind speeds 10 units above the median wind speed (Equation 4.3), and precipitation values below a user defined limit (Equation 4.3). The rules below describe data elements that satisfy the e-commerce example described above.

$$
\begin{aligned}
\text { currentbid } & >\operatorname{avg}(\text { currentbid })+\text { stddev }(\text { currentbid }) \\
\text { time } & >\frac{9}{10} * \max (\text { time })
\end{aligned}
$$

Sometimes the user might search for elements whose attributes satisfy combinations of several rules. Users can employ boolean AND and OR operations to tie rules together or specify their independence from other rules. Also, rules may make use of constants and common statistical tools such as standard deviation, average, median, max, and min. These functions provide users with the necessary tools to create meaningful sets of rules.

\subsection{Areas of Interest}

Once the user has constructed a set of EOIs to study, the next step is to incorporate them into coherent structures. By spatially clustering EOIs, users define regions of potential interest within their datasets. This simplifies the exploration tasks by allowing users to more easily identify regions they should focus on from the rest of the dataset. We assume the size of the set

of EOIs is much smaller than $n$. A large set of EOIs usually implies that the user's concept of 
interesting is either too general or too vague. As an oversimplified example, if a user specifies interesting elements as those in which a specified attribute is greater than its median, one half of the dataset is now considered interesting.

Even though the size of the set of EOIs is small compared to $n$, the number of EOIs can overwhelm a display in one of two ways. Small fractions of large datasets may still contain hundreds of data elements, making it difficult to fit all of the EOIs on-screen. Likewise, EOIs could be spread out over a large spatial area, making it impossible for the user to simultaneously view all elements of interest in detail.

By "clustering" the EOIs based on their spatial positions, we attempt to alleviate the users' burden of locating and navigating to EOIs outside their current view. By displaying spatial clusters of EOIs in a high-level overview display, the user can more easily identify the presence and general location of the EOIs. An overview display allows a user to navigate to a location that contains a cluster. Within this local region, clusters establish boundaries, or borders, that users may need to determine how they will explore the surrounding area. Clustering the EOIs also divides the dataset into regions and districts as described by Lynch, creating a visualization that supports efficient mental map creation [Lyn60]. The geometric properties of clusters also contribute to the visualization by providing additional survey and landmark knowledge to the user. 


\subsection{Constructing Areas of Interest}

Once the EOIs have been identified, they must be organized into areas of interest, or AOIs. The AOIs are built using a spatial clustering algorithm that takes a set of EOIs as input and returns the set of EOI clusters as output. The basic clustering algorithm is described below:

1. Let $A_{j}$ be an empty set.

2. Choose $e_{i}$ from the set $E$ of EOIs as a starting point and add it to $A_{j}$.

3. Select the set of elements $E_{\text {point }}$ from $E$ that are within a threshold $\delta$ of $e_{i}$, and add them to $A_{j}$.

4. Compute the convex hull, $C_{j}$, of $A_{j}$.

5. Select the set of elements $E_{\text {hull }}$ from $E-A_{j}$ that are within the threshold $\delta$ from the boundary of $C_{j}$ and add them to $A_{j}$.

6. Re-compute $C_{j}$, and repeat the selection process until there are no elements within distance $\delta$ of the convex hull or there are no more points in $E$.

The algorithm returns a partition of $E$ into EOIs that belong to $A_{j}$ and those that do not. Subsequent AOIs may be built by applying the same algorithm to the EOIs that remain in $E-A_{j}$

The above algorithm represents a simple and effective clustering technique, but it require some extensions to work properly in our large dataset environment. Grouping EOIs that are spatially close to one another is only one way to structure the shape of an AOI. Users might 


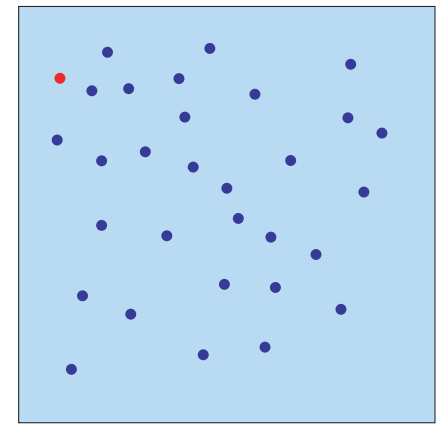

(a)

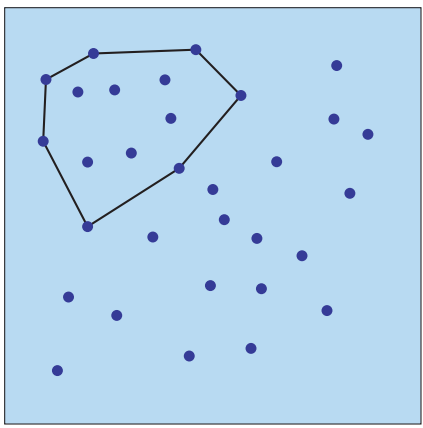

(d)

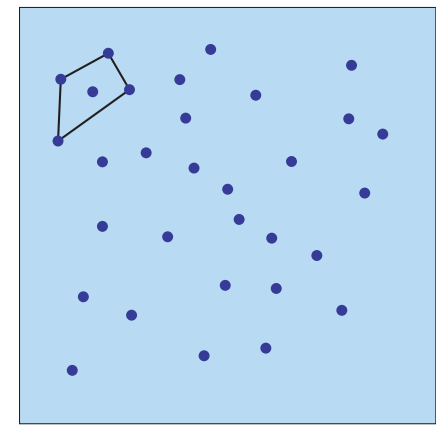

(b)

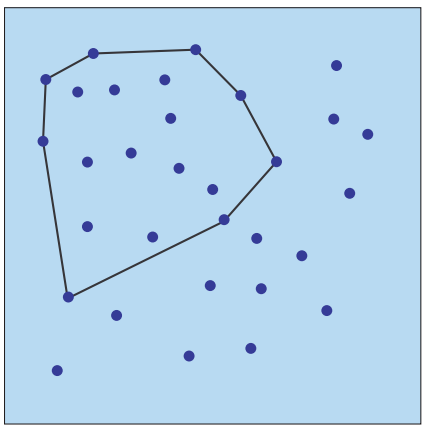

(e)

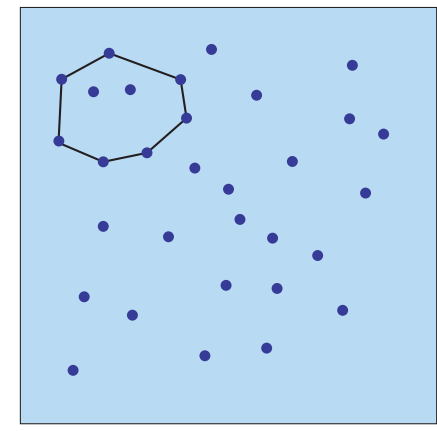

(c)

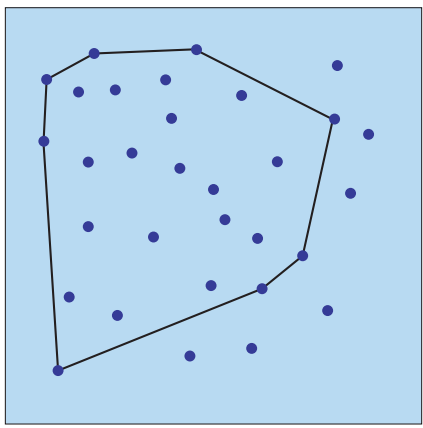

(f)

Figure 4.1: An example of how the convex hull algorithm constructs a cluster. The initial vertex is colored red in (a). (b)-(f) shows how elements within $\delta$ of the convex hull are identified and added to $A_{j}$ until the distance from the convex hull to all remaining vertices is greater than $\delta$

want to influence the clusters in other ways. This can be accomplished by considering additional parameters during AOI construction.

\subsubsection{Proximity}

The proximity parameter is used to control the physical size and density of AOIs. This parameter represents $\delta$ in the primary clustering algorithm. High proximity values will allow for a smaller number of large AOIs. Low proximity values will generate a larger number of small AOIs. 


\subsubsection{Population}

The population parameter places an upper bound on the logical size of an AOI by specifying the maximum number of EOIs that can be contained within a single AOI. Specifying a small population will partition a set of EOIs into a larger number of smaller AOIs. This means the user navigates smaller sets of data elements, resulting in a possible reduction in the number of AOIs whose data elements cannot be viewed simultaneously. However, the user must now manage a larger overall number of AOIs in order to explore the entire set of EOIs.

\subsubsection{Area}

The area parameter places an upper bound on the physical size of an AOI. Physically smaller AOIs may be easier to manage since the user needs to keep track of less off-screen information. However, users must again explore a larger number of AOIs to view every EOI.

The area of a convex hull can be computed by forming a triangulation of the hull. The simplest triangulation for a convex hull of $n$ vertices is to select an arbitrary vertex $v$ on the convex hull and form an additional $n-3$ edges by connecting $v$ to every other hull vertex not

adjacent to it. The area of the convex hull can be found by computing the sum of the areas of each triangle formed by the additional edges. 


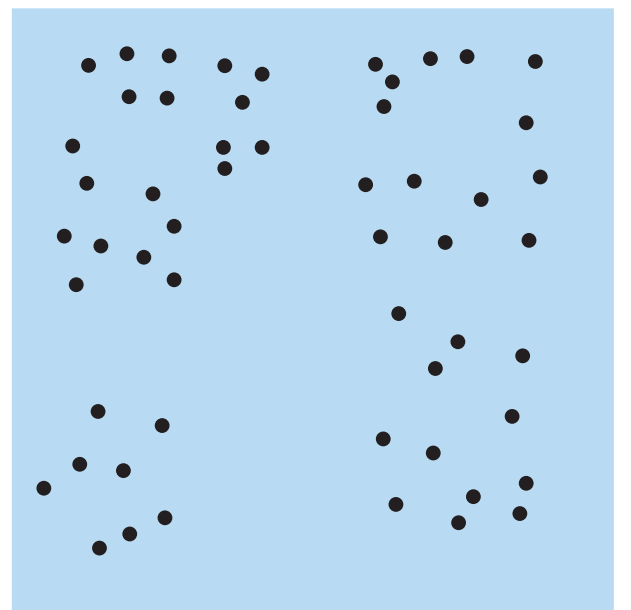

(a)

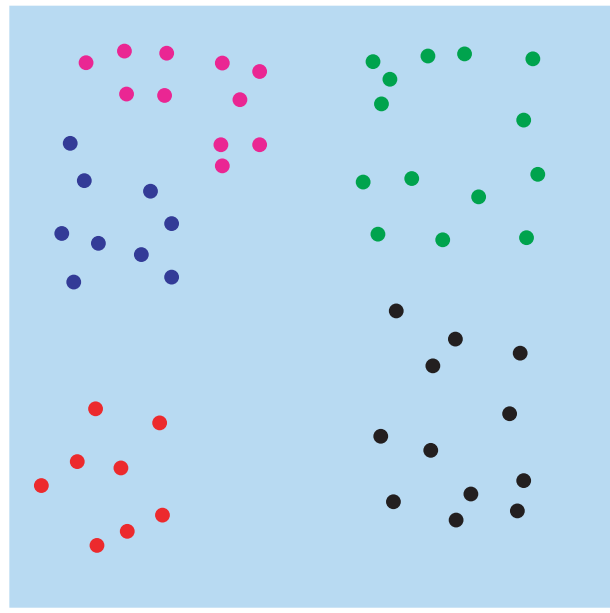

(c)

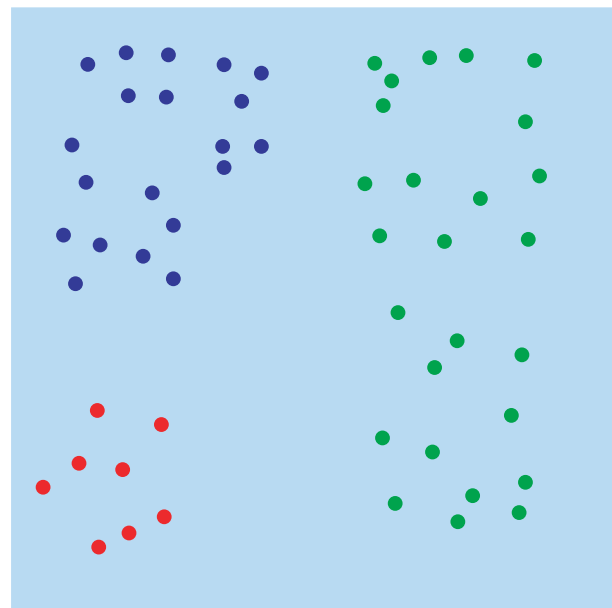

(b)

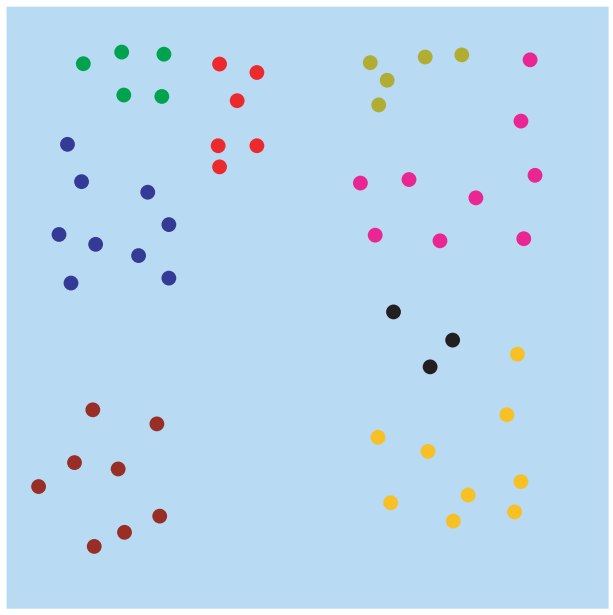

(d)

Figure 4.2: An example of how specifying the parameters population, proximity, and area influence the structure of a cluster: (a) A cluster formed with a population $=60$, proximity $=3$, and area $=50$; (b) shows the cluster in (a) with proximity changed to 1 ; (c) shows the cluster in (a) with area changed to 5; and (d) shows the cluster in (a) with population changed to 10 . 


\section{Chapter 5}

\section{Graphs of the Navigation Framework}

Once the areas of interest have been constructed, they are integrated into the navigation framework. The framework serves three purposes. First, it provides visual cues to the user about the locations of elements of interest within the information space. Viewers use these cues to aid with navigation decision making. Second, the framework also provides levels of imageability as described by Lynch [Lyn60]. Increased imageability aids users by preserving their sense of position within the environment. Third, the framework provides a data structure that can be used by the navigation assistant to implement a variety of automated tours through the data.

The framework is structured using two types of graphs: local and global. Graphs are an ideal instrument to use for the framework. They are a flexible structure that is easily integrated into a virtual environment. Graph traversal algorithms provide a solid foundation for camera planning. The global graph links the AOIs together, providing a unified structure of AOIs. The

global graph is represented with a Euclidean minimum spanning tree built from the centers of 
each AOI. This graph is meant to assist users with global movement between AOIs. A local graph is built within each AOI to assist with navigating between the AOI's elements of interest. We chose a Delaunay triangulation of EOI positions to construct the local graph. Each of these local graphs is connected to the minimum spanning tree to complete the framework.

\subsection{Discussion of Relevant Graph Theory}

For many of the following sections, we employ the following graph theory and definitions. A graph $G$ is composed of a set of vertices, $V(G)$, and a set of edges, $E(G)$, where every edge $e \in E(G)$ is represented by an unordered pair of distinct vertices in $V(G)$. For every $e$, there exists $u, v \in V(G)$ such that $e=u v$ or $e=v u$. A graph is a weighted graph if every edge $e \in E(G)$ is assigned a numeric value denoted as $w(e) . w(e)=w(u, v)$ where $e$ connects the vertices $u$ and $v$. Our system makes use of weighted graphs with non-negative values. All of the graphs used in the navigation assistant are simple graphs, e.g. graphs that do not contain loops or multiple edges which share the same two endpoints.

A complete graph is a graph constructed by connecting every vertex to every other vertex. A planar graph is a graph whose vertices and edges can be laid out in a plane such that no two edges will cross. The dual graph of $G$ is a graph $G^{*}$ which has a vertex $v^{*}$ for every face of $G$, i.e. if $F_{i}$ is a face of $G$, then there exists $v_{i}^{*} \in V\left(G^{*}\right)$. For every edge $e \in E(G)$, with $e$ adjacent to the faces $F_{i}$ and $F_{j}$, there exists an edge $e^{*} \in E\left(G^{*}\right)$ formed by connecting the the vertices $v_{i}^{*}$ and $v_{j}^{*}$ in $G^{*}$. The dual of a planar graph is also planar. A straight-line dual is the dual of a graph whose edges are all lines. 


\subsection{Voronoi Diagrams and Delaunay Triangulations}

Given a set $S$ of $n$ points in the plane, for each point $p_{i} \in S$ what is the set of points $(x, y)$ in the plane that are closer to $p_{i}$ than to any other point in $S$ [PS85]? The solution to this problem is a partitioning of the plane into polygons, $P_{i}$, some of which are unbounded, where $P_{i}$ represents the locus of points $(x, y)$ closest to the given point $p_{i}$ in $S$.

For any two points, $p_{i}, p_{j} \in S$, we can partition the plane into two half-planes. One halfplane represents the locus of points $(x, y)$ closest to $p_{i}$, while the other half-plane contains the points closest to $p_{j}$. The half-plane containing $p_{i}$ and all points in the plane closer to $p_{i}$ than to $p_{j}$ is denoted as $H\left(p_{i}, p_{j}\right)$. Such a half-plane is defined by the perpendicular bisector of the line segment $\overline{p_{i} p_{j}}$.

The locus of points $(x, y)$ closest to $p_{i}$ can be described as the intersection of $n-1$ halfplanes. This intersection forms a polygonal region called the Voronoi polygon associated with $p_{i}$, denoted $V(i)$.

$$
V(i)=\bigcap_{i \neq j} H\left(p_{i}, p_{j}\right)
$$

The $n$ Voronoi polygons corresponding to the $n$ points of $S$ form the Voronoi diagram of $S$, denoted $\operatorname{Vor}(S)$. Vertices in the Voronoi diagram are called Voronoi vertices and edges of the Voronoi diagram are called Voronoi edges. In a Voronoi diagram, if $(x, y)$ is contained in $V(i)$, then $(x, y)$ is closer to $p_{i}$ than to any other point in $S$.

The dual of a Voronoi diagram is called a Delaunay graph. The straight-line dual of a Voronoi diagram is a triangulation of the points in $S$. This graph has many properties that 


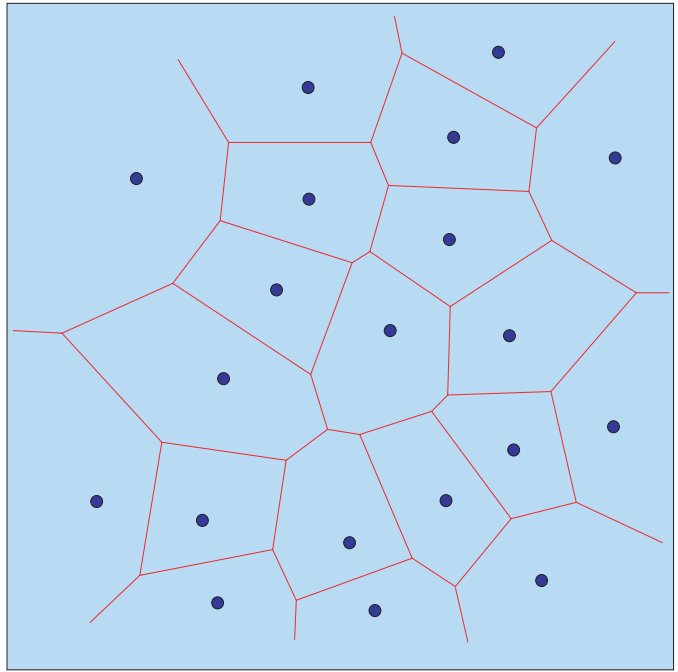

(a)

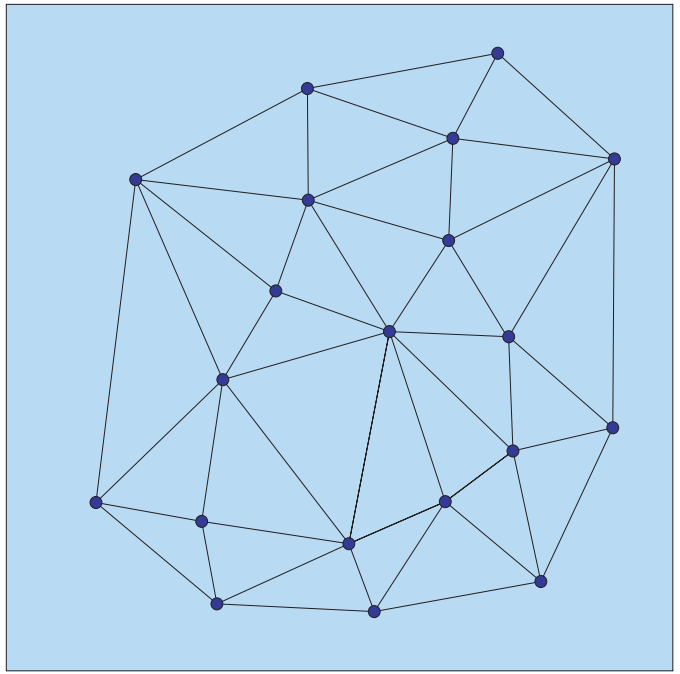

(b)

Figure 5.1: A Voronoi diagram is shown in (a) and its corresponding Delaunay graph is shown in (b).

make it an ideal choice to be used for the local graphs of our framework.

For an evenly distributed set of points, the corresponding Delaunay triangulation avoids long, thin triangles, using full triangles constrained to a local area within the set of points. The Delaunay graph is a planar graph. There exist polynomial-time graph traversal algorithms for planar graphs. The Delaunay triangulation has a number of edges linear with respect to the number of points in $S$. Euler's formula for planar graphs states that a planar graph has no more than $3 N-6$ edges [Wes01]. This restriction prevents an explosive increase of the number of edges for a large set of points. Likewise, it is shown that the average degree of a vertex in the Delaunay graph is at most six. 


$$
\begin{aligned}
\sum_{v \in V(G)} \operatorname{deg}(v) & =2 e \\
\sum_{v \in V(G)} \operatorname{deg}(v) & \leq 2(3 N-6) \\
\frac{\sum_{v \in V(G)} \operatorname{deg}(v)}{N} & \leq \frac{6 N-12}{N} \\
\operatorname{avg}(\operatorname{deg}(v)) & \leq 6
\end{aligned}
$$

Therefore, Delaunay graphs typically have low branching factors. The Delaunay graph provides edges that help to visualize spatial relationships between EOIs within an area of interest. The Voronoi polygon for a data element represents the spatial neighborhood of that data element. Users are often located within the neighborhood of the element they are currently visualizing. To view a set of EOIs, users typically move between adjacent neighborhoods. A Delaunay edge is created between two points when their neighborhoods are adjacent. Therefore, the Delaunay graph provides directional information to neighboring EOIs that are adjacent to the current EOI. This property makes Delaunay graphs a useful tool for visualizing the spatial relationships between EOIs.

\subsection{Euclidean Minimum Spanning Tree}

Like the local graphs, the global graph is meant to provide specific navigation aids. We expect users will need to move between AOIs, especially if they are spread throughout the dataset. We use a simple spanning tree structure to connect the set of AOIs. 


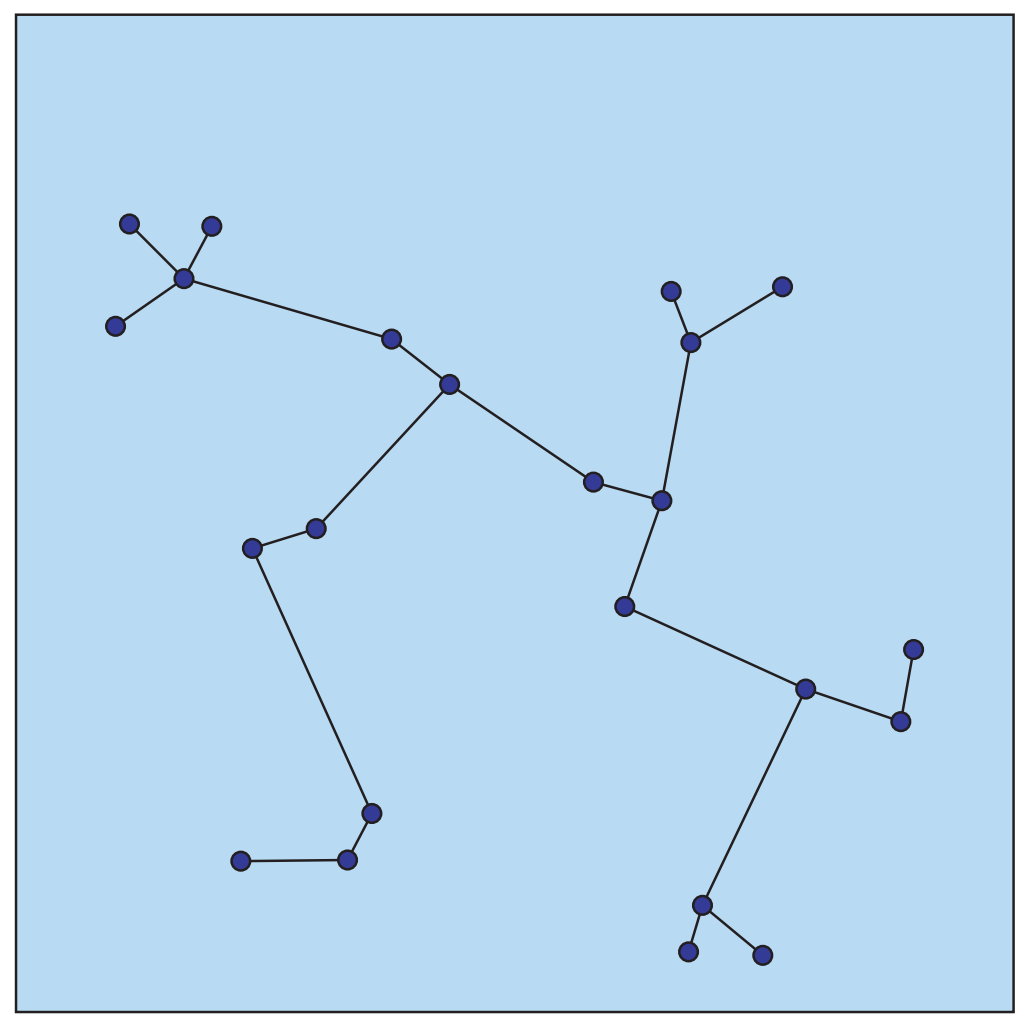

Figure 5.2: The Euclidean minimum spanning tree for a set of points.

A Euclidean minimum spanning tree was chosen to act as the global graph because of the particular set of edges it contains. The minimum spanning tree is constructed with Kruskal's algorithm using the Euclidean complete graph whose vertices are the centers of the set of AOIs. The spanning tree is initialized by adding every vertex as an individual component. Each edges is assigned a weight equal to its Euclidean length. Iterating through the edge list from smallest to largest weight values, an edge is added to the tree when it connects two disjoint components. Components can be existing subtrees or disjoint vertices. Each edge added to the spanning tree decreases the number of disjoint components by one. The algorithm terminates when there exists a single component. 
Since Euclidean distances are used for the edge weights, Kruskal's algorithm connects each AOI to its closest neighbor. The algorithm connects AOIs spatially near to one another early in its execution, ensuring short paths that produce clusters of AOIs. The same connectivity feature occurs between the clusters. The result is a Euclidean minimum spanning tree that supports efficient global navigation, since the edges of the tree connect spatially neighboring AOIs. 


\section{Chapter 6}

\section{Assisted Navigation}

With the graph framework in place, the navigation assistant has the ability to perform various types of exploration tasks for the user. The assistant provides these operations by generating automated tours based on the user's requests. An automated tour is built in three steps. First, using a graph algorithm, an ordered sequence of EOIs is created for the user to view. Second, the navigation assistant computes an optimal viewpoint from which to display each EOI. Finally, a spline curve is formed through the viewpoints, completing the construction of an automated camera path.

\subsection{Graph Traversal Algorithms}

In addition to providing a visualization of the spatial relationships between EOIs, the local graph is used as a spatial data structure by the navigation assistant. The first step in building a camera path is to determine which elements of a dataset to visit and in what order they 
should be seen. Using a set of traversal algorithms on the framework's graphs, the assistant can generate a variety of ordered EOI sequences. An ordering is important because it defines the exploration path through the dataset to satisfy the user's navigation request. The assistant currently implements two types of graph traversals: shortest path and minimum Hamiltonian cycle approximation. The graph theory of each traversal will be described before its application.

\subsubsection{Dijkstra's Algorithm}

A common problem in graph theory is computing the shortest path between two vertices in a graph. An $x, y$-path is a sequence of edges and vertices, $\left\{v_{0}, e_{1}, v_{1}, e_{2}, v_{2}, \ldots, e_{n}, v_{n}\right\}$, where the endpoints of $e_{i}$ are the vertices $v_{i-1}$ and $v_{i}, v_{0}=x$, and $v_{n}=y$. No edge occurs more than once in an $x, y$-path. The distance, $d(x, y)$, for an $x, y$-path in a weighted graph is the sum of the edge weights of the edges along the $x, y$-path. For any two distinct vertices $x$ and $y$ in a graph $G$, we often want the $x, y$-path for which $d(x, y)$ is minimal.

Dijkstra's algorithm can be used to find the minimal (or shortest) $x, y$-path in $O\left(V^{2}+E\right)$, where $V$ is the number of vertices and $E$ is the number of edges in $G$. The algorithm works by incrementally building a list of vertices, $S$, whose shortest path from a given source vertex, $x$, has been computed. Let $\delta(v)$ be the current estimated cost of the shortest $x, v$-path. Initially, $\delta(v)=\infty$ for all $v \neq x$. For all $v_{i}$ adjacent to $x, \delta\left(v_{i}\right)$ is updated with an initial estimate (i.e. $\left.w\left(x, v_{i}\right)\right)$. A vertex $v_{j} \notin S$ with the minimum $\delta\left(v_{j}\right)$ is then selected and added to $S$. Then vertices $v_{k} \notin S$ and adjacent to $v_{j}$ have their estimates updated. If $\delta\left(v_{k}\right)>\delta\left(v_{j}\right)+w\left(v_{j}, v_{k}\right)$, then $\delta\left(v_{k}\right)$ is updated to $\delta\left(v_{j}\right)+w\left(v_{j}, v_{k}\right)$ to reflect the new, shorter path through $v_{j}$. When 


\title{
DIJKSTRA'S ALGORITHM
}

\author{
Initialization \\ $\mathrm{D}[\mathrm{x}]=0$ \\ for $\mathrm{v} \in \mathrm{V}(\mathrm{G})$ and $\mathrm{v} \neq \mathrm{x}$ do \\ $\mathrm{D}[\mathrm{v}]=\mathrm{w}(\mathrm{xv})$ \\ $\mathrm{P}[\mathrm{v}]=\mathrm{x}$ \\ $\mathrm{S}=\{\mathrm{x}\}$
}

\section{Iteration}

while $\mathrm{S} \neq \mathrm{V}(\mathrm{G})$ do

select $\mathrm{v} \notin \mathrm{S}$ such that $\mathrm{D}[\mathrm{v}]=\min (\mathrm{D}[\mathrm{v}])$ for all $\mathrm{v} \notin \mathrm{S}$

$\mathrm{S}=\mathrm{S} \bigcup\{\mathrm{v}\}$

for every vertex y adjacent to $\mathrm{v}$ do

if $\mathrm{D}[\mathrm{y}]>\mathrm{D}[\mathrm{v}]+\mathrm{w}(\mathrm{vy})$ then

$\mathrm{D}[\mathrm{y}]=\mathrm{D}[\mathrm{v}]+\mathrm{w}(\mathrm{vy})$

$\mathrm{P}[\mathrm{y}]=\mathrm{v}$

\section{Algorithm 1: Pseudocode for Dijkstra's algorithm.}

complete, Dijkstra's algorithm computes the shortest path from source vertex $x$ to every other vertex in $V(G)$.

The pseudocode in Algorithm 1 shows the details of Dijkstra's algorithm. Given source vertex $x$ the algorithm maintains an array $D$ such that $D[i]$ is the length of the shortest known $x, v_{i}$-path, or $\delta\left(v_{i}\right)$. For any two vertices $u, v \in G$ where $G$ is a weighted graph, $w(u, v)=\infty$ if no edge connects $u$ to $v$.

The array $P$ is used to backtrack along the shortest path from $x$ to any other vertex in $G$. $P[v]$ holds the vertex that precedes $v$ on the shortest path from $x$ to $v$. An example of Dijkstra's algorithm is given in Figure 6.1.

The application of a shortest path is apparent. Shortest paths provide the least expensive traversal between vertices in our AOI graphs. Although a user might be able to move more 


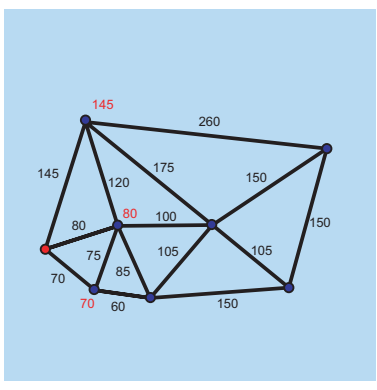

(a)

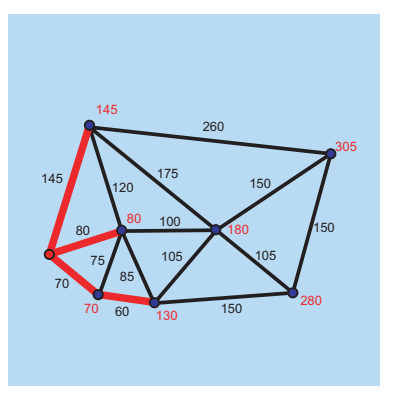

(e)

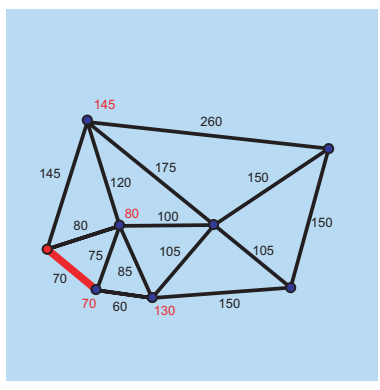

(b)

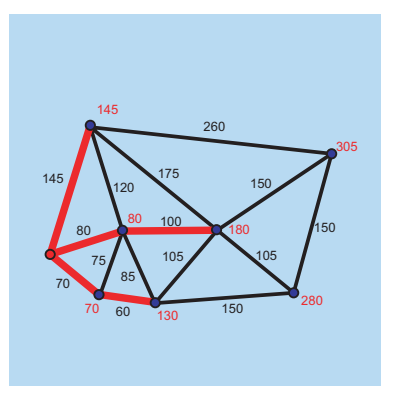

(f)

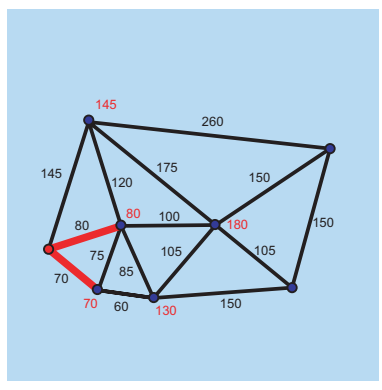

(c)

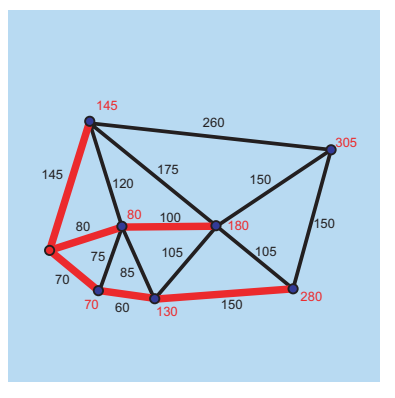

(g)

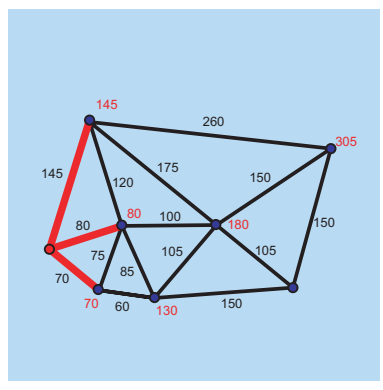

(d)

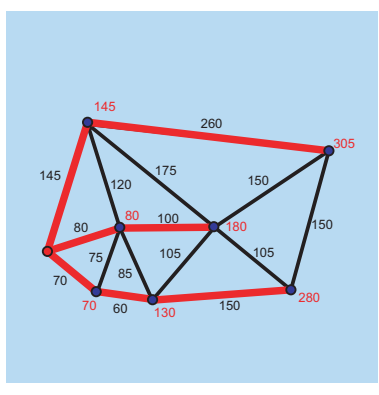

(h)

Figure 6.1: An example of Dijkstra's algorithm computing the shortest paths from the vertex in red to all other vertices. A red number adjacent to a vertex $v$ corresponds to the value $\delta(v)$.

quickly by ignoring the graph and simply navigating directly between the two vertices, this type of straight line motion is not necessarily advantageous for exploration. The shortest paths generated using the graph framework provide relatively short tours that allow users to view additional elements of interest between the start and end positions. Paths can also be created between EOIs from different AOIs by using the centers of AOIs as vertices of $G$. In this way, the assistant performs tradeoffs between the amount of time spent exploring the dataset against the amount of potentially useful information shown to the user. 


\subsubsection{Hamiltonian Graphs and the Traveling Salesman Problem}

A cycle of a graph is a $u, v$-path where $u=v$. A spanning cycle is a cycle that contains every vertex in the graph. A graph is called Hamiltonian if it contains a Hamiltonian cycle, a cycle with no duplicate edges that visits every vertex exactly once. Determining whether a general graph is Hamiltonian is an NP-complete problem.

Finding a Hamiltonian cycle is similar to determining the minimum cost Hamiltonian cycle, also know as the Traveling Salesman problem (TSP). Practical applications of this problem are numerous. Since our system uses Euclidean distances for the weights of edges, we would like to find a polynomial algorithm to build a minimal cost Hamiltonian cycle. Unfortunately, finding Hamiltonian cycles in Delaunay triangulations has also been shown to also be NPcomplete [GGJ76, PS76, Pap77].

Since a solution to the Traveling Salesman problem cannot be found in polynomial time, we chose to employ an algorithm that approximates the solution. We take advantage of the fact that the edges of our Delaunay triangulation obey the triangle inequality. The triangle inequality is a mathematical relation that states that the length of the sum of components is greater than or equal to the sum of the lengths of components.

$$
|x+y| \leq|x|+|y|
$$

For a triangle, this relation implies that the sum of the lengths of any two of its edges is greater than or equal to the length of its third edge. Because of this property, a minimum spanning tree of $G$ (computed in $O(E \lg E$ ) time [Wes01]) can be used to rapidly approximate 
the minimum cost Hamiltonian cycle.

Using a minimum spanning tree, we can approximate a tour that is no more than twice the cost of the true minimal Hamiltonian cycle [RSL77]. The algorithm begins by computing the minimum spanning tree of $G$. Then, every edge of the tree is doubled. This creates a graph that is Eulerian. In an Eulerian graph, every vertex has even degree, that is, every vertex is adjacent to an even number of edges. Every Eulerian graph has a spanning cycle (an Euler cycle) [Wes01]. After the Euler cycle is built, an initial vertex is arbitrarily chosen to start a traversal of the cycle. Vertices are added to the approximated tour in the order they are encountered on the Euler cycle. During the traversal, if a vertex is seen that has already been visited, it is skipped. Since the graph edge weights obey the triangle inequality, we know that a direct path from the current vertex to the next unvisited vertex is always less expensive than a path that includes additional edges from the Euler cycle.

Let $\Phi$ denote the edges of the minimal cost Hamiltonian cycle of $G$, and let $T$ represent the minimum spanning tree of $G$. Removing an edge $e$ from $\Phi$ creates a path which is also a spanning tree. By the definition of a minimum spanning tree, $\operatorname{cost}(T) \leq \operatorname{cost}(\Phi-e)<$ $\operatorname{cost}(\Phi)$. Constructing an Euler cycle by doubling every edge of the minimum spanning tree is equal to $2 * \operatorname{cost}(T)$ which implies that the approximation is less than $2 * \operatorname{cost}(\Phi)$.

Computing approximations of the solution to the Traveling Salesman problem creates complete tours of an AOI, allowing users to visualize all the EOIs in the AOI. While these approximations are not necessarily the shortest cycles available, they allow the assistant to rapidly construct a complete tour that attempts to minimize the distance traveled. Another use of this 


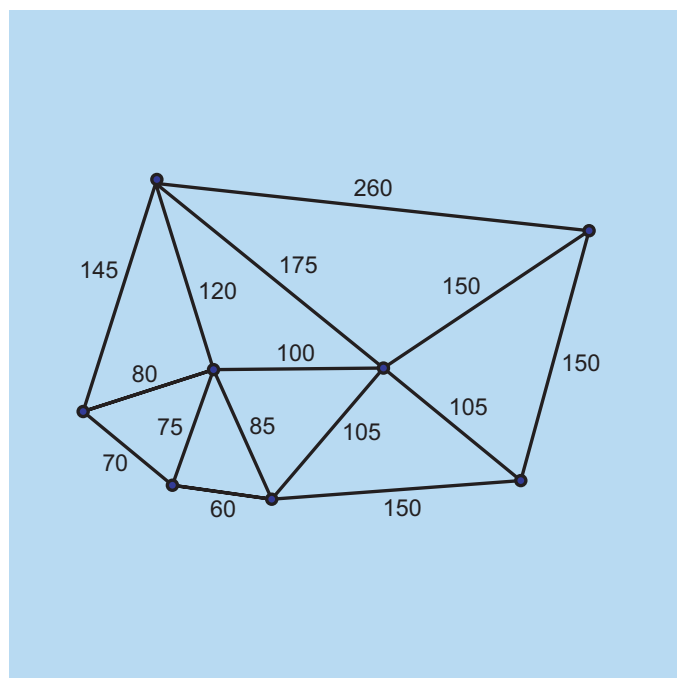

(a)

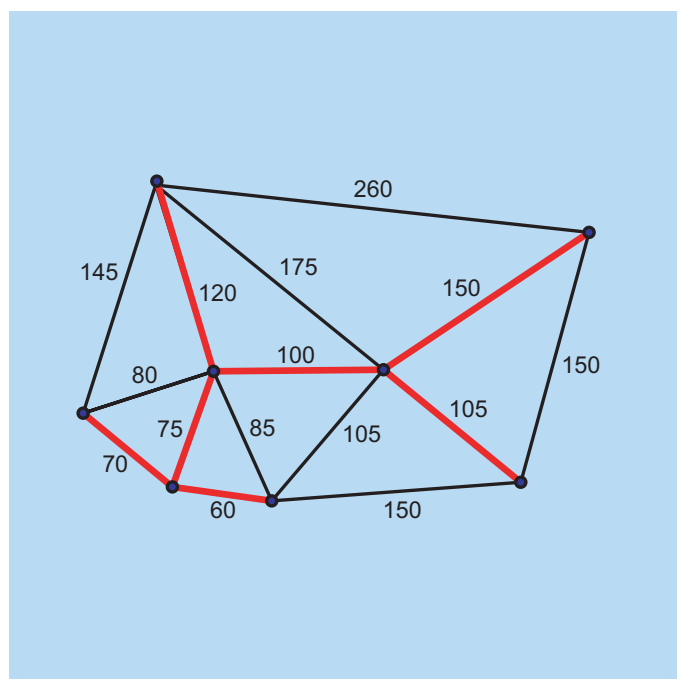

(c)

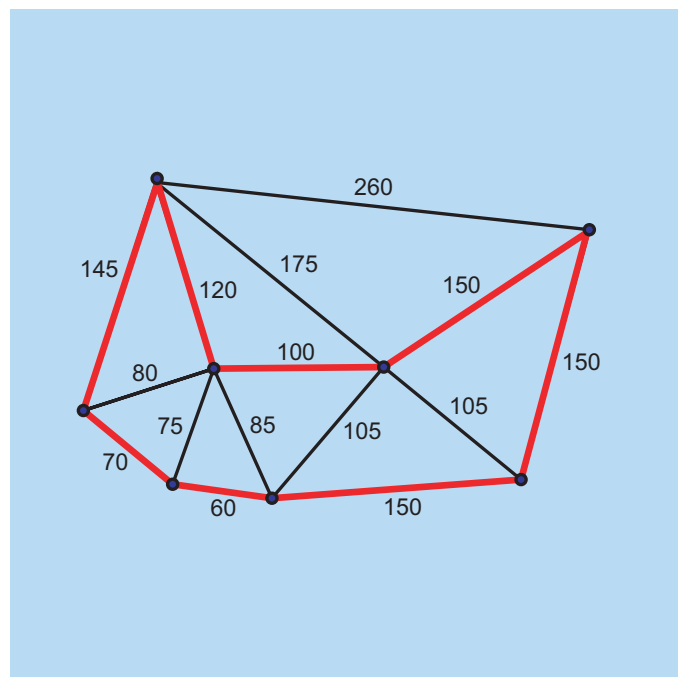

(b)

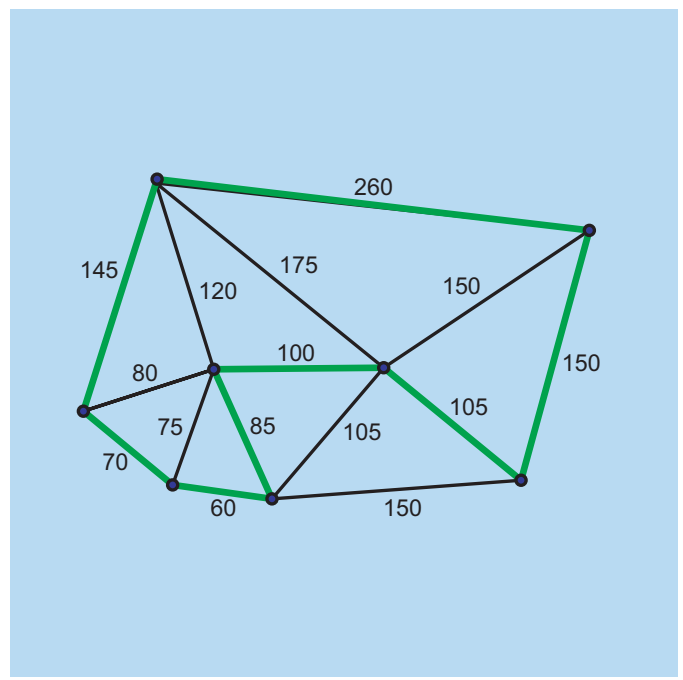

(d)

Figure 6.2: (a) is a graph whose edge weights obey the triangle inequality. (b) shows the minimal Hamiltonian cycle of this graph (cost of 945). (c) shows the minimum spanning tree used to build the approximation of the minimal Hamiltonian cycle (cost of 975) in (d) 
algorithm is computing a tour of between the centers of the AOIs (without visiting their individual EOIs).

\subsection{Optimal Viewpoints}

Once an ordered sequence of EOIs has been constructed, we must select a camera location from which to view each EOI. The optimal viewpoint is the camera position that provides the "best" view of the EOI in question. Mathematically, this viewpoint can be specified relative to an EOI using spherical coordinates, $(r, \theta, \phi)$. Let $v$ be the vector drawn from a point $p$ to the origin. The spherical coordinates of $p$ are defined as follows: $r$ is the length of $v, \theta$ is the angle formed between $v$ and the $x$-axis, and $\phi$ is the angle formed between $v$ and the $z$-axis. Users might want small $r$ values to be able to closely examine EOIs, or large $r$ values to obtain contextual views. Users need the freedom to specify preferences for optimal viewpoints in order to meet their exploration goals. The navigation assistant will consider these preferences together with other constraints (e.g. avoiding viewpoints where the EOI is occluded or avoiding viewpoints whose view direction is too low relative to the EOI) to select the final optimal view position for each EOI.

Automated camera planning has been studied by a variety of researchers [wHCS96, DZ94, BL99]. Bares and Lester used a constraint solver to determine an optimal viewpoint for an object with a set of viewing constraints. If a viewpoint could not satisfy the constraints, the system computed alternative views by relaxing certain constraints until an optimal viewpoint was found. This concept is appealing, because it provides an efficient and flexible tool for cam- 
era planning. Also, Bares and Lester's constraint designs are not confined to a specific visual environment. The camera position that satisfies the maximum set of constraints is accepted as optimal.

Our system uses a constraint solver similar to Bares and Lester's. Our navigation assistant needs only a small number of constraints to define optimal viewpoints. Three basic constraints were used to define an optimal viewpoint:

1. Occlusion: The ability to see an object without visual interference from neighboring elements of the visualization. This constraint is especially important when exploring visualizations containing elements with varying heights.

2. View angle: The view angles are the $(\theta, \phi)$ directions from the EOI to the camera. They represent which side of the EOI the camera is pointed towards and how much the camera looks down on the EOI.

3. View distance: The $r$ component of the camera position (relative to the EOI) in spherical coordinates. This constraint represents how far away the camera is from the EOI.

The constraint solver gives precedence to occlusion over the viewing parameters, because it was deemed more desirable for the user to be able to actually see the target than to preserve a preferred view direction. This means we relax the view angle and distance constraints (if necessary) to meet the occlusion constraint.

Regions of space that satisfy the view angle constraints are projected onto the hemisphere centered at the EOI and lying on the world plane. A camera is then placed at the EOI and 


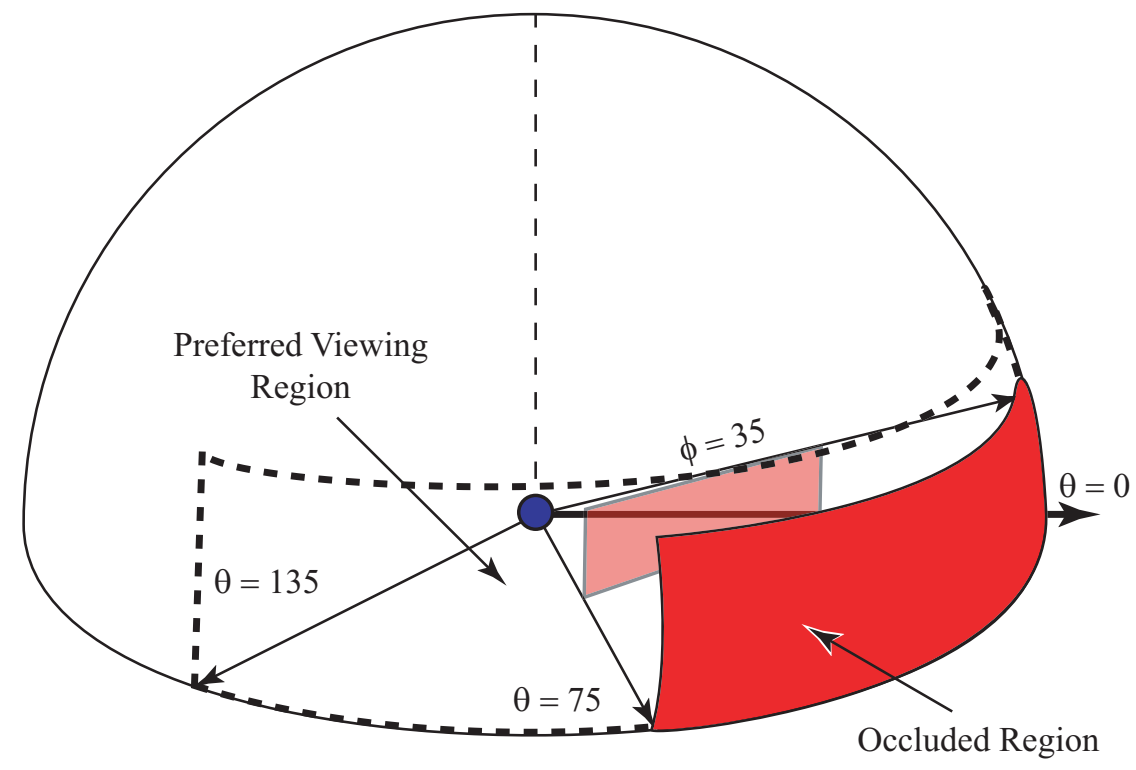

(a)

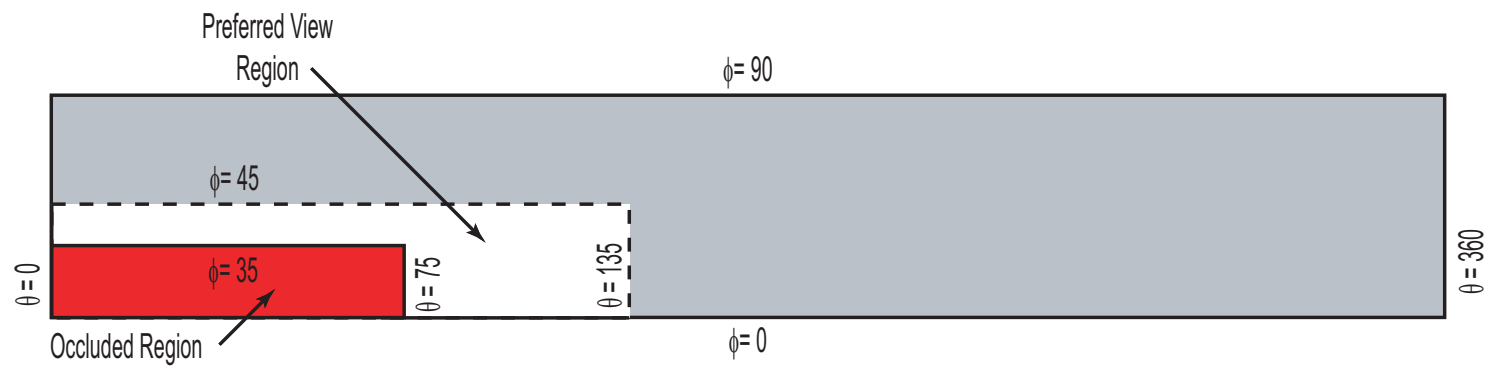

(b)

Figure 6.3: (a) shows an occluded region formed by projecting an object onto a hemisphere. The user's projection of the user's preferred viewing region is also shown. (b) shows how the occlusion and preferred view regions are represented in a buffer. 
used to project all objects visible from the EOI on to the hemisphere. This marks out occlusion regions. If the intersection of non-occluded regions and preferred view angles is non-empty, there exists view positions that satisfy both the view angle and the occlusion constraints. If there are no such regions, the view angle constraint is relaxed. Figure 6.3 demonstrates the process of projecting occlusion regions onto a hemisphere.

The projections are implemented using bitmaps and bitwise AND operations to search for intersections. Two buffers are constructed: one to represent view angle constraints, and another to represent occlusion regions. Building the viewing constraints buffers is trivial; areas that satisfy view constraints can be marked directly in the buffer.

The occlusion hemisphere is built by taking five snapshots of the world from a camera translated to the EOI's position. The perspective angle of the camera is $90^{\circ}$. The five shots capture a north, east, south, west, and up view. For the initial shot, the camera is placed at the EOI pointing down the $x$-axis. The camera is rotated $90^{\circ}$ about the $z$-axis to capture subsequent shots, until all $360^{\circ}$ have been seen. Since the camera's perspective angle is $90^{\circ}$ and the camera is lying on the plane, the pyramid region directly above the EOI has not been recorded. This region is captured by aiming the camera along the $z$-axis. The combinations of these five shots represent the hemisphere centered at the EOI.

Once the buffers have been constructed, the search for an intersection is performed. Corresponding bytes in the viewing buffer and occlusion buffer are bitwise AND-ed to search for an intersection. From the viewpoints found which satisfy both buffers, the viewpoint closest to the center of the preferred viewing region is selected for the optimal viewpoint. If there exists 


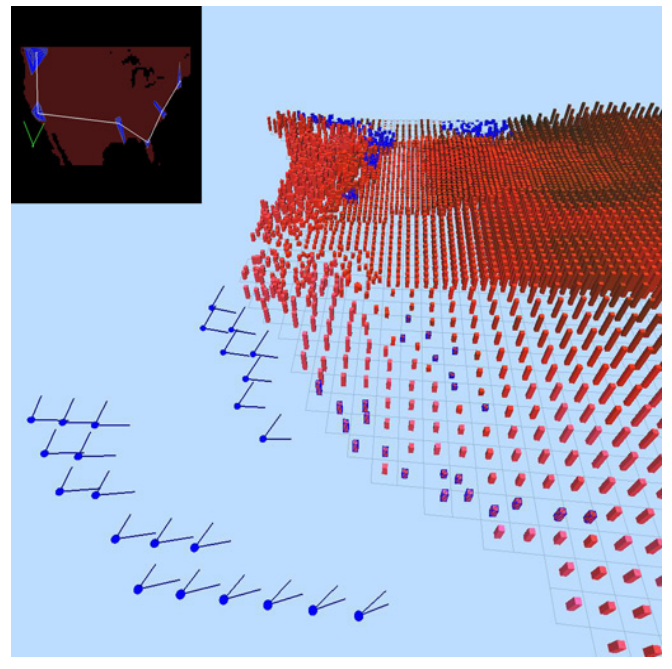

(a)

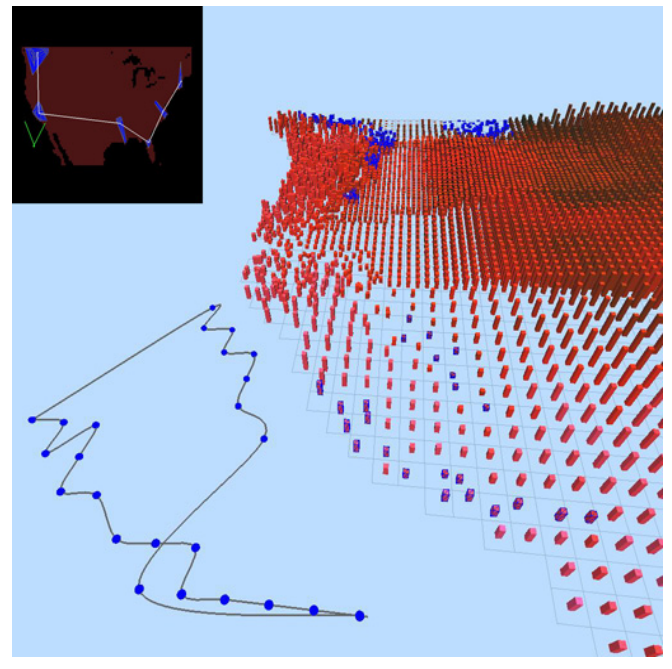

(b)

Figure 6.4: (a) is a set of optimal viewpoints. The camera's view and up vectors are shown for each viewpoint. (b) shows a spline path that passes through each point.

no intersecting region, the view angle constraint is relaxed by doubling the view angle region in the corresponding buffer. Once the optimal viewpoint for each EOI has been determined, the system can proceed to build a path between each optimal viewpoint. This path will be used by a camera to visit viewpoints.

\subsection{Camera Path}

Given the set of optimal viewpoints, a camera path must be constructed that will visit each view position in a way that gives users effective views of each EOI. The camera must transit from one viewpoint to another in a manner that allows the user to maintain a sense of position in the visualization. The camera should move fluidly though the visualization, providing appealing shots of EOIs and smooth pans from one EOI to another. 
A camera animation is made up of a sequence of shots. A shot is composed of three values: a viewpoint, a view vector, and an up vector. The viewpoint is a camera position in three dimensional space. The view vector is the vector from the viewpoint to the direction that the camera is aimed. The up vector is the vector that defines that "up" direction for the camera.

\subsubsection{Quality Cinematic Shots}

In an attempt to build appealing animations, the assistant models its camera paths on the work of cinematographers. The field of cinematography has been studied since the advent of motion pictures. Cinematographers want to produce visually coherent camera shots in an artistic manner. Previous work has investigated techniques to emulate cinematographers [wHCS96, DZ94, BL99]. Creating a comprehensive set of rules to simulate a cinematographer is a difficult task. Cinematography is an art form that requires careful consideration of the specific goals and the context of each camera shot. Different cinematographers exhibit unique styles, and many techniques are often improvised for a particular situation. It is unlikely that there exists a set of cinematographic rules for an automated camera planner that will always produce high quality shots. However, there are common guidelines that do an excellent job of producing good shots for a wide range of common camera planning environments. These rules can be used to build camera paths to produce effective animations:

1. The camera's alignment with the world's up vector should remain constant. This prevents the camera from twisting around on its view axis. Favoring a consistent up direction helps users maintain their orientation within the visualization. 
2. The camera's view should be focused on the subject of the shot. If the camera is not focused on the subject, viewers do not know what they are supposed to be looking at. It is often an assumption that whatever is located in the center of a view is the object of importance.

3. Camera motion and pans should be smooth. Rough panning and jerky camera motion hampers users' abilities to reorient themselves when the view changes.

4. The camera should not collide with objects in the scene. Instead of moving through an object, the camera should pass around it.

Each of these issues is considered when we construct the animation to view a set of EOIs. The methods used to follow these guidelines are discussed below.

\subsubsection{The Camera Path and Spline Curves}

The first step in building the camera path is to locate the locus of points the camera should follow. The path should be continuous and smooth. It needs to start at the viewer's location and move in a fluid fashion to every EOI's optimal viewpoint. Moreover, the camera path should provide shots that will satisfy the guidelines from Section 6.3.1. These conditions motivated the use of a curve for the path of the camera. Creating curves between a set of points is a classic problem in mathematics called interpolation.

By far the most common solution to interpolating a curve from a discrete set of points is to use spline curves. A spline curve is a set of polynomial functions that are connected together 
by continuity conditions. Typically, these polynomials are of degree three and have the form:

$$
S(t)=a+b t+c t^{2}+d t^{3}
$$

The set of points $\left\{p_{1}, \ldots, p_{n}\right\}$ to be interpolated by a spline curve are known as the curve's control points.

Spline curves are normally a composition of multiple curve segments. How these segments are connected is specified by a set of continuity conditions. These conditions define the "smoothness" of the spline curve at its join locations. Continuity can be described mathematically by considering the limits of a function $f$.

$$
\lim _{x \rightarrow a^{-}} f(x)=\lim _{x \rightarrow a^{+}} f(x)=f(a)
$$

As values converge to $a$ from below $(x<a), f(x)$ will approach $f(a)$. Likewise, as values converge from above ( $x>a), f(x)$ will approach $f(a)$. Mathematically, this determines the smoothness of a curve. Spline functions that meet the above condition are called $C_{0}$ continuous (i.e. the end points meet at a common spatial position). Curves are $C_{n}$ continuous if they satisfy:

$$
\lim _{x \rightarrow a^{-}} f^{(n)}(x)=\lim _{x \rightarrow a^{+}} f^{(n)}(x)=f^{(n)}(a)
$$

Curves used for camera paths should satisfy specific continuity conditions to maintain smooth animation. A curve used for camera paths must be connected at the join points. There- 


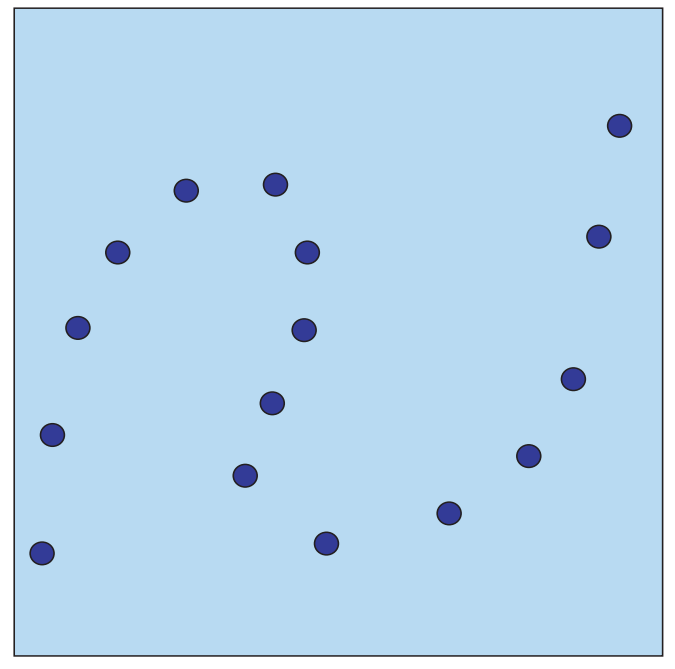

(a)

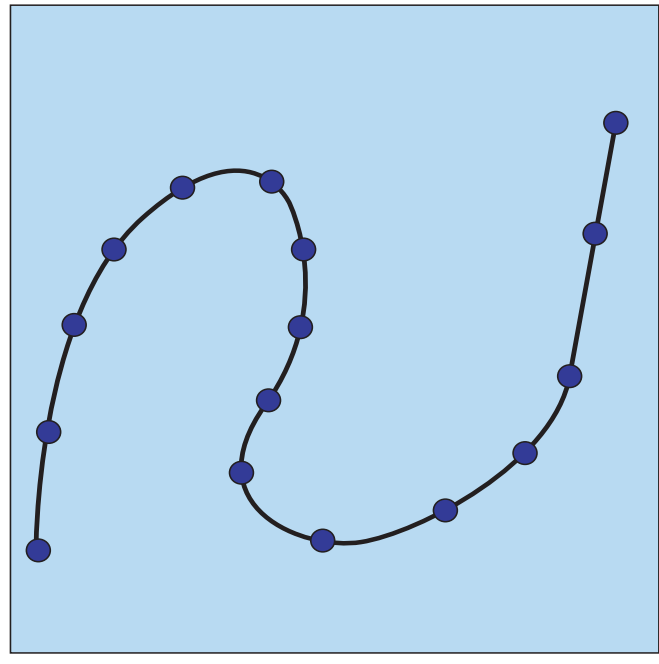

(b)

Figure 6.5: (a) is a set of control points and (b) shows a natural spline curve that fits these points

fore, it must be $C_{0}$ continuous. As a camera moves along a curve, its velocity and acceleration along the curve should be continuous to maintain smooth movement. Therefore, the curve must also be $C_{1}$ and $C_{2}$ continuous. Finally, the camera paths of our system must pass through each optimal viewpoint to guarantee an effective view of every EOI. Therefore, the spline curve must pass through each of its control points. Interpolation functions that contain their control points are known as exact interpolations.

There are a variety of spline curves, but only a few satisfy the required continuity conditions and provide an exact interpolation. The natural spline is an exact interpolation that is $C_{0}, C_{1}$, and $C_{2}$ continuous. The natural spline of $n$ control points is composed of $n-1$ curve segments. Finding the polynomials that represent each segment requires computing the coefficients of Equation 6.2. Mathematically, this is accomplished by solving a system of linear equations. Solving a general system of equations can be done with Gaussian elimination in $O\left(n^{3}\right)$ time 
[CK99, BF01]. However, the structure of a natural spline's system of equations allows the solution to be computed in linear time, $O(n)$ [Ebe01]. Therefore, natural splines provide an effective camera path curve that can be computed rapidly and efficiently.

\subsubsection{Maintaining Effective Exploration and Preserving Quality Shots}

Given a natural spline camera path with appropriate continuity that passes through our optimal view positions, we next turn to the problem of controlling the camera's up vector and avoiding collision with objects in the scene. We preserve the up vector for a shot by checking the angle between the world up vector and the view vector. A large angle implies that the camera is looking directly down on its subject. Low angle views are preferred over high angle views. If a large angle exists between the vectors we insert extra control points to try to reduce it.

Avoiding collision is accomplished in a similar fashion. As each curve segment is built, the system checks for collision with elements along the camera path. If a collision is discovered, the curve is raised until it passes over the element in question. Once all collision points have been identified, a camera path is constructed that monotonically rises to the necessary height and then smoothly descends back to the next optimal viewpoint. Raising the camera can potentially violate the user's preferred viewing angle, but the system treats this constraint as less important than providing a collision-free path.

Keeping the proper subject in the center of the view and panning smoothly is accomplished by constructing a view vector based on a spline curve's parametric value $t$. For a given spline segment $S_{i}$ which passes through optimal viewpoints $e_{i}$ and $e_{i+1}$, consider a re- 
parameterization of its domain $\left[t_{i}, t_{i+1}\right]$ to $[0,1]$. For the interval, $\left[0, \frac{1}{3}\right)$ the assistant's camera focuses on $e_{i}$. For the interval $\left[\frac{1}{3}, \frac{2}{3}\right)$, the camera pans smoothly from $e_{i}$ to $e_{i+1}$. For the remaining interval $\left[\frac{2}{3}, 1\right]$, the camera focuses on $e_{i+1}$. If $T_{i}$ is the time needed to traverse the curve segment, $S_{i}$, the camera will be focused on EOI $e_{i}$ for $\frac{1}{3} T_{i-1}$ (corresponding to the camera's approach) and $\frac{1}{3} T_{i}$ (corresponding to the camera's departure from $e_{i}$ ). This technique could potentially cause the occlusion of $e_{i}$ during a portion of the animation. However, passage through the optimal viewpoint guarantees a non-occluded view of $e_{i}$ during some part of the time period that the camera is aimed at $e_{i}$. Also, any occlusion that does occur will smoothly appear and disappear as the camera moves toward the optimal viewpoint. This manner of constructing view vectors creates smooth panning and prioritizes aiming the camera at a relevant subject during the time the camera is not panning (for $e_{i}$, the camera pans for $\frac{1}{3} T_{i}$ ).

\subsubsection{User Control}

Once the camera path is complete, the navigation assistant begins its animation. Even though the assistant manipulates the camera during an animation, the user is able to re-acquire control at any time. In data exploration, the interests of a user can change suddenly and unexpectedly. Seeing new elements might cause users to re-evaluate their goals. A user could change their focus of attention to different properties of the data or decide to spend more time studying a particular element. Because it is impossible to always predict the user's needs, the navigation system is specifically designed not to be fully automated. 


\section{Chapter 7}

\section{Practical Applications}

We acquired a collection of weather data to test the navigation assistant in a real-world setting. This dataset uses surface climate readings from the years 1961 to 1990 to compute monthly

average values at $\frac{1}{2}^{\circ}$ latitude and longitude increments for all surface positions throughout the world. For each sample point, the mean value of each attribute is computed for each month. This dataset contains a total of $m=7$ values: mean temperature (temp), wind speed (wind), cloud coverage (cloud), precipitation (precip), month, latitude, and longitude.

Perceptual texture elements (or pexels) that look like rectangular towers were used to visualize the weather data for a given month. Along with color, recall that pexels use the texture properties height, density, and regularity to visualize multiple attributes at a single location. Our visualization maps temp to color: dark blues and greens for low temperatures to bright reds for high temperatures; wind to height: taller pexels for higher wind speeds; cloud to density: high densities for heavier cloud cover; and precip to regularity: irregular pexels for high 
precipitation. For example, tall, densely packed dark blue and dark green pexels to cold regions with dense cloud cover and high wind speeds (Figure 7.1a).

The following rules were used to define elements of interest:

1. $\operatorname{precip}>\operatorname{avg}($ precip $)+\operatorname{stddev}($ precip $) \& \&$ cloud $<\operatorname{avg}($ cloud $)-\frac{1}{2}$ stddev $($ cloud $)$

2. $\operatorname{precip}>\operatorname{avg}($ precip $)+\operatorname{stddev}($ precip $) \& \&$ wind $>\operatorname{avg}($ wind $)+\frac{1}{2}$ stddev $($ win $)$

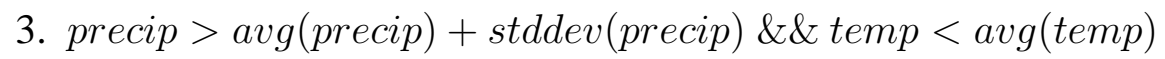

The above rules identify regions of high precipitation that contain secondary weather conditions of interest to the viewer (e.g. high wind speeds and low temperatures), or that are unexpected (e.g. low cloud cover). When the rules are applied to the dataset, EOIs are discovered across the visualization and segmented into six AOIs (Figure 7.2). EOIs are outlined in blue for easy visual identification. Figure 7.3 shows a tour built for a large AOI in the Pacific Northwest. This AOI is composed of EOIs representing high precipitation coupled with low cloud cover or high wind speed.

A global overview of the entire dataset is provided in the upper-left corner of the screen to assist users with a variety of tasks (Figure 7.1b). The overview provides no detailed information about individual data elements, but it effectively visualizes the location and size of the AOIs. The global graph structure is also visualized in the overview, offering visual cues about the spatial relationships of AOIs. The viewer's position and field of view is shown in the overview as a view frustum to locate the viewer within the dataset and to help him to maintain that location during animation sequences. 


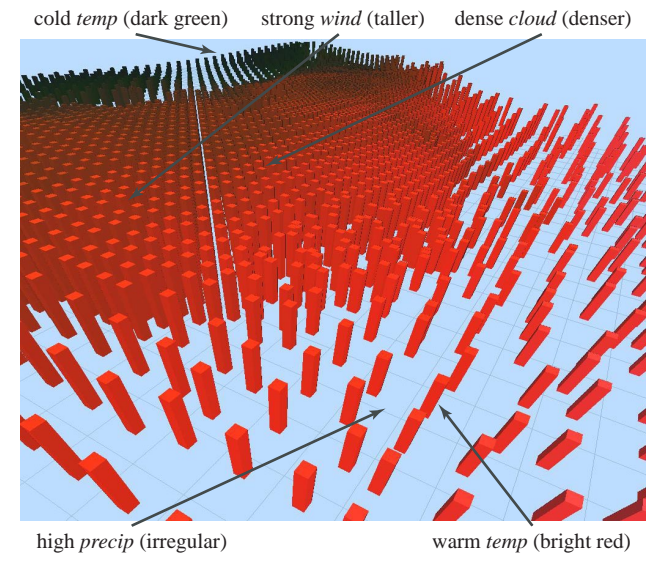

(a)

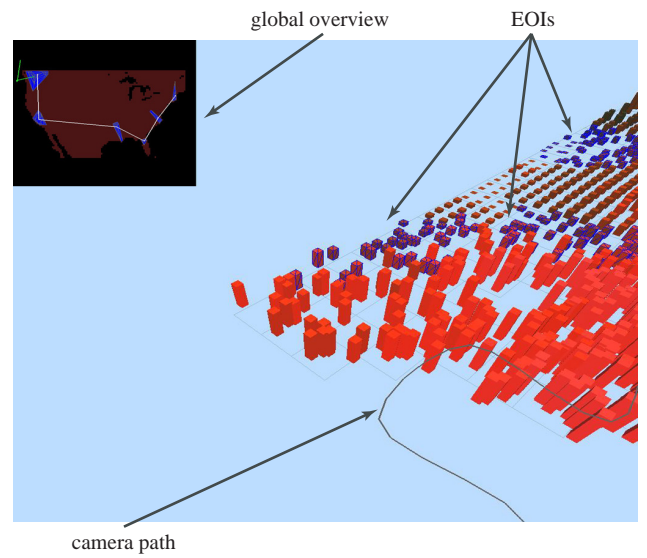

(b)

Figure 7.1: The visualization of weather data for the Pacific Northwest region is shown in (a). (b) is a screen shot showing highlighted EOIs, a camera path, and the overview.

Figure 7.3 shows a collection of camera views taken from an automated tour of the AOI located in the Pacific Northwest. The camera path is shown in light gray. Note how the camera starts in the northwestern-most corner of the United States and proceeds eastward to reach the optimal viewpoints for the eastern-most EOIs in this AOI. The camera moves south to visualize the southern EOIs, before returning to the northwestern-most area where the tour began.

This is an example of just one type of automated tour available. Users may request navigation assistance between individual EOIs belonging to a large AOI. Figure 7.4 demonstrates the tour from one EOI of a large cluster to another EOI located on the opposite side of the AOI. The camera adjusts its path to view EOIs that would have been omitted on a linear path. Users can also generate tours that navigate from one AOI to another. Figure 7.5 shows a sequence of shots taken from a tour originating at one EOI in the Pacific Northwest to an EOI located on the other side of the visualization, the Eastern seaboard. As it approaches AOIs on this tour, the camera focuses on their centers, providing users with views of other regions of interest. All 
of these tours are constructed by using the graph framework to generate a sequence of optimal viewpoints. A smooth camera path is established between these points to view relevant EOIs and AOIs during the animation process. 


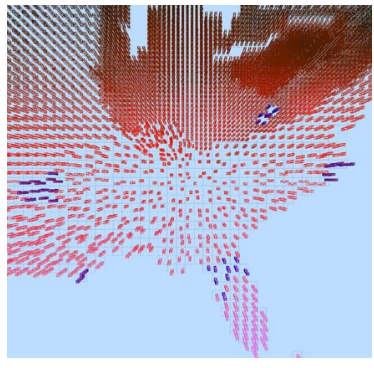

(a)

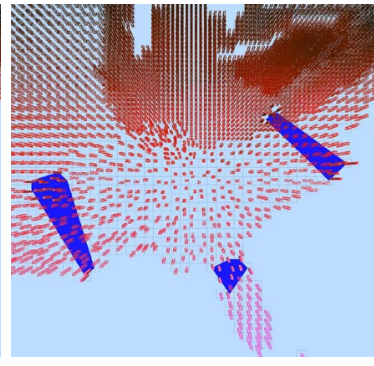

(b)

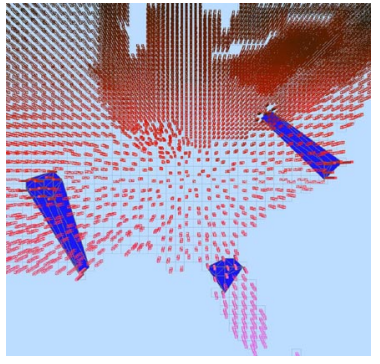

(c)

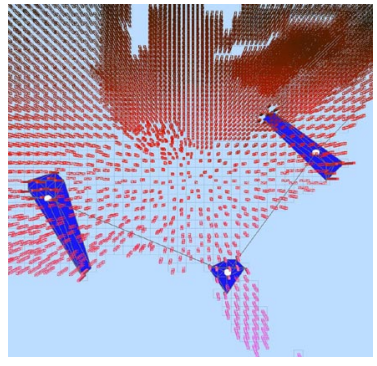

(d)

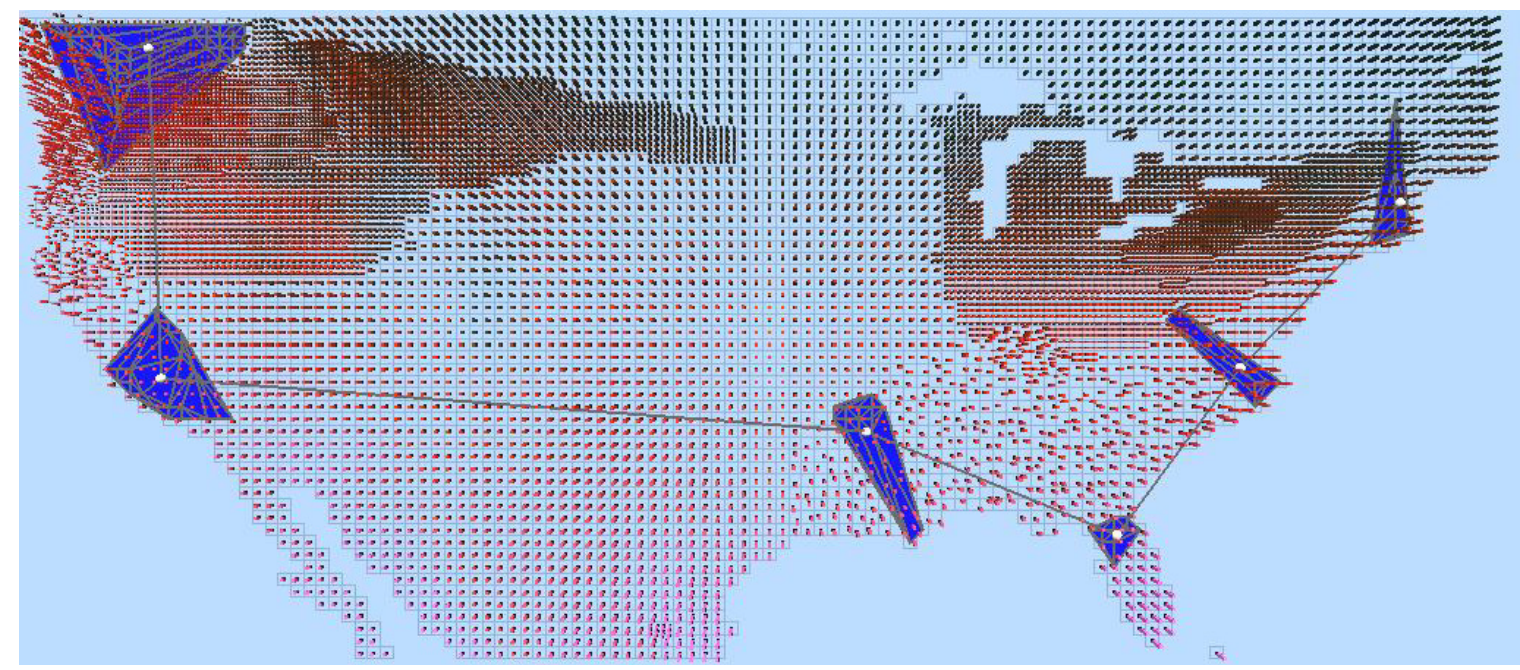

(e)

Figure 7.2: Areas of interest constructed in an example weather dataset: (a) individual elements of interest (shown as shaded solids with highlighted borders) are selected via user specified rules; (b) the elements are spatially clustered into areas of interest; (c) elements within each area are connected with a Delaunay triangulation; (d) areas of interest are connected with a minimum spanning tree; (e) a global view of six areas of interest within the continental United States 


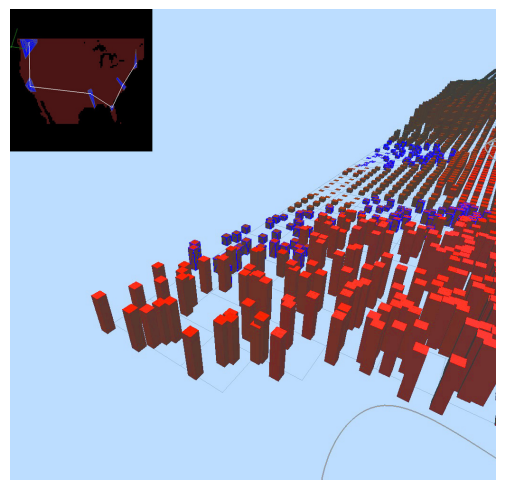

(a)

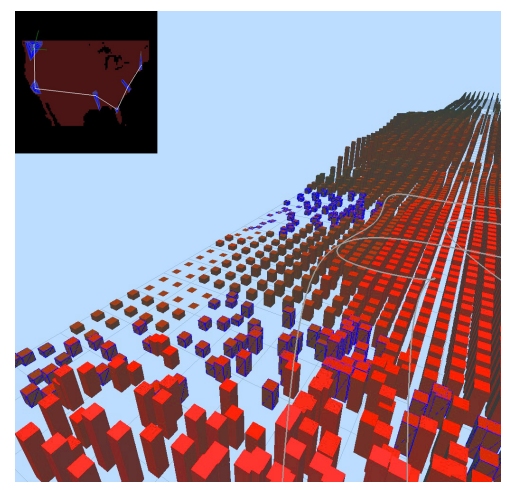

(d)

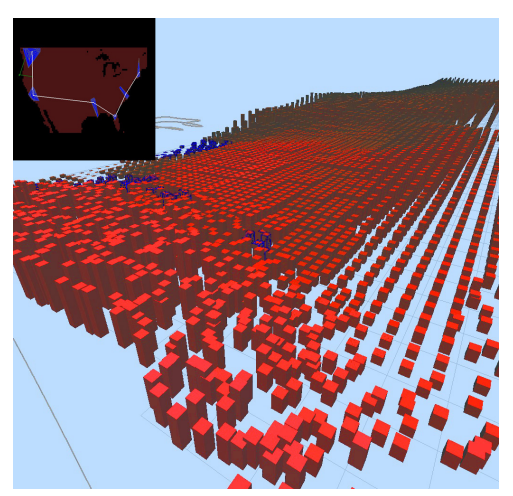

(g)

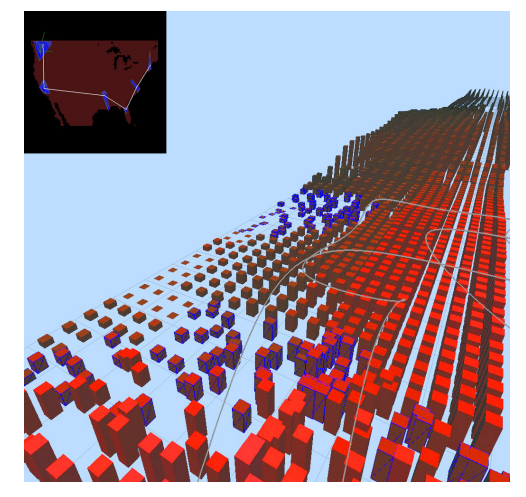

(b)

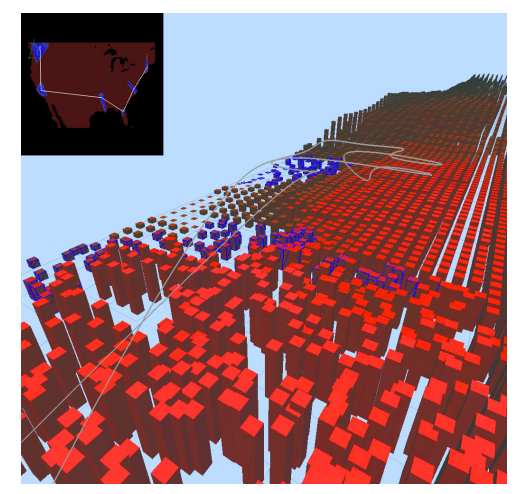

(e)

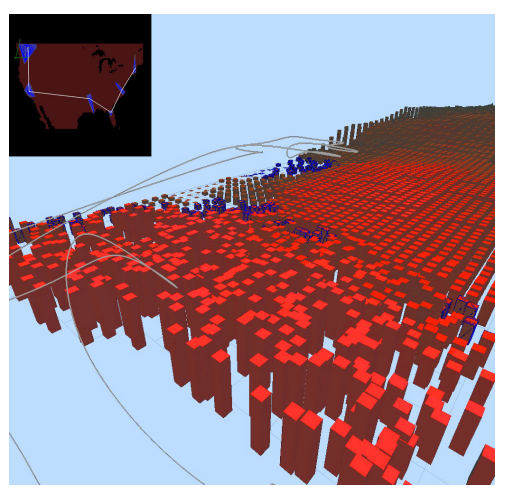

(h)

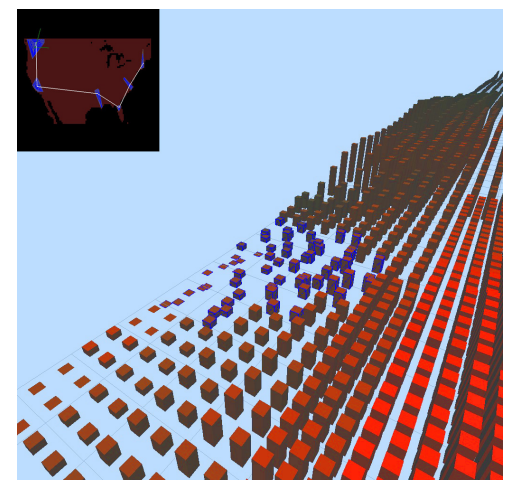

(c)

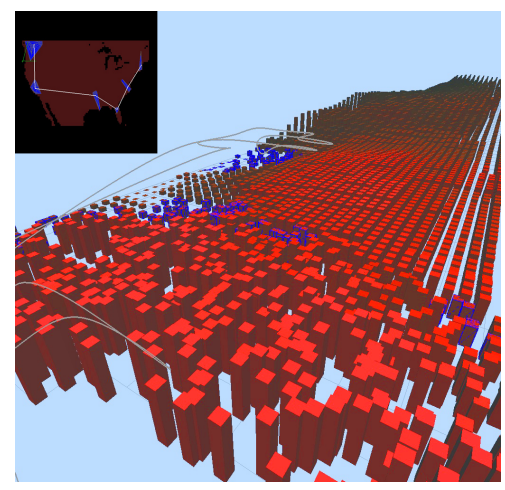

(f)

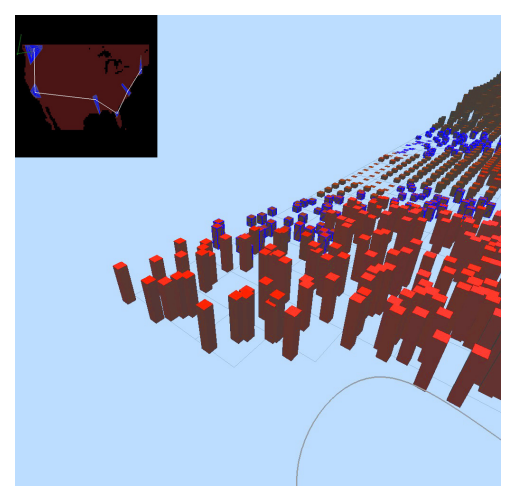

(i)

Figure 7.3: (a)-(c) depicts the camera moving from the western EOIs to the eastern EOIs. (d)-(g) shows the camera moving south to explore more of the AOI. (h) and (i) display the camera returning to the starting point. 


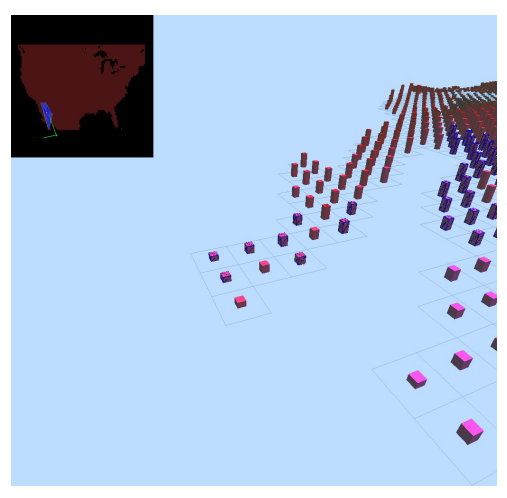

(a)

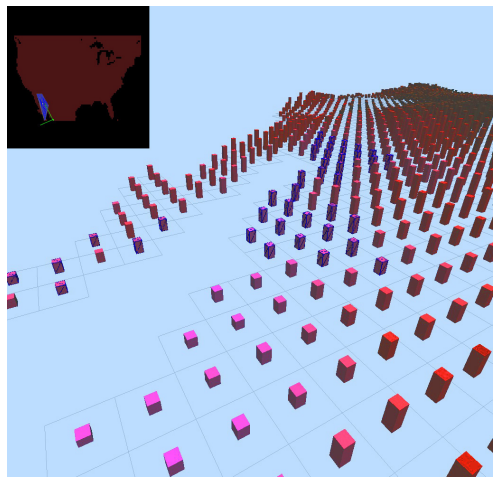

(c)

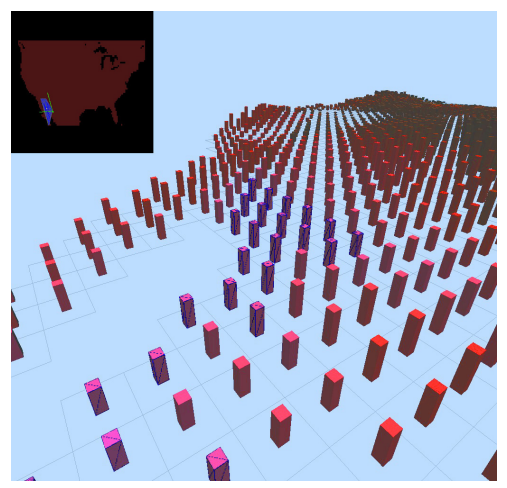

(e)

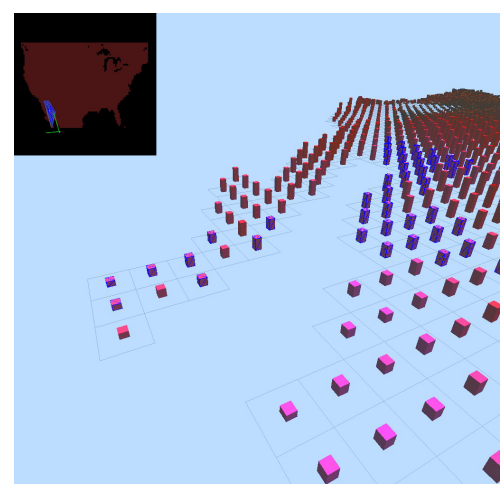

(b)

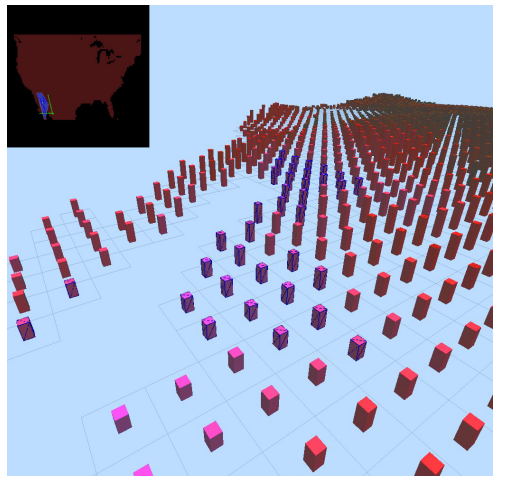

(d)

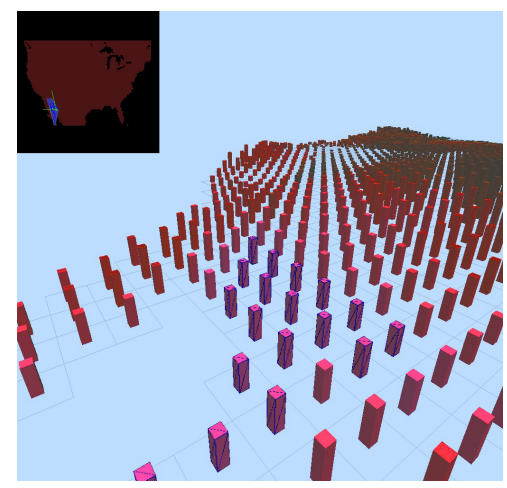

(f)

Figure 7.4: An example of a shortest path tour of an AOI. Instead of moving from the southernmost EOI to the northwestern-most EOI, the camera detours to view additional EOIs in the northeastern corner of the AOI. 


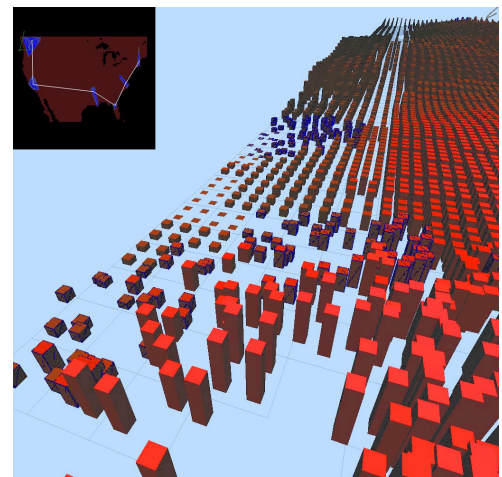

(a)

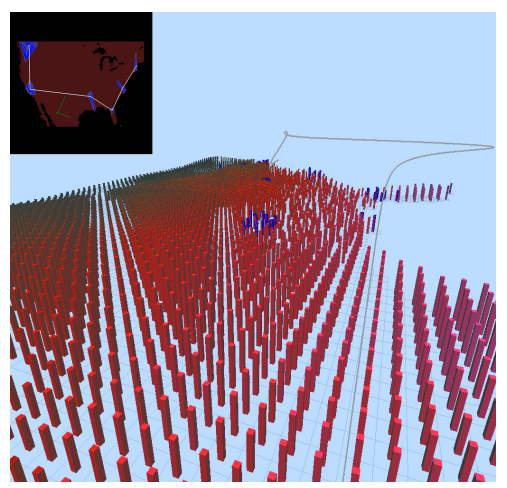

(d)

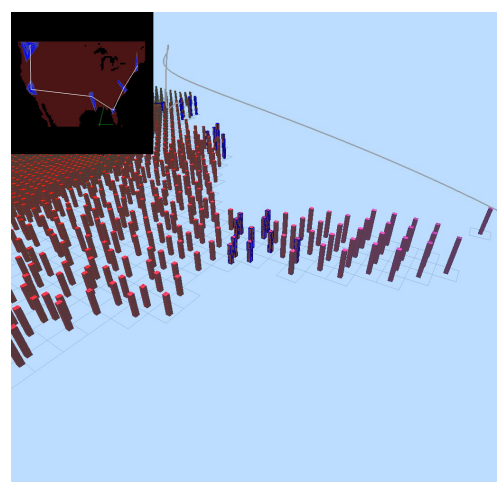

(g)

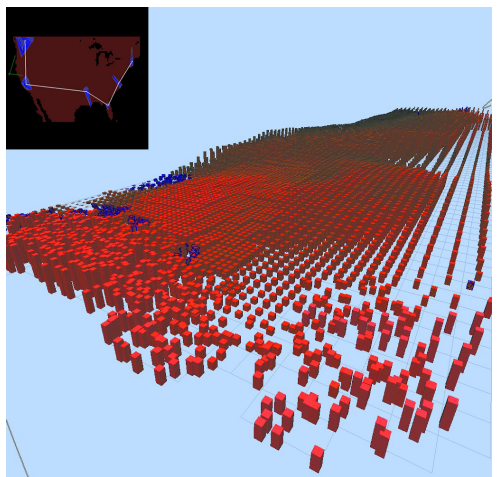

(b)

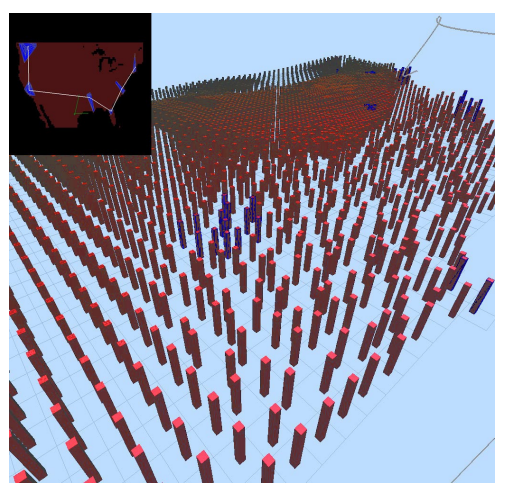

(e)

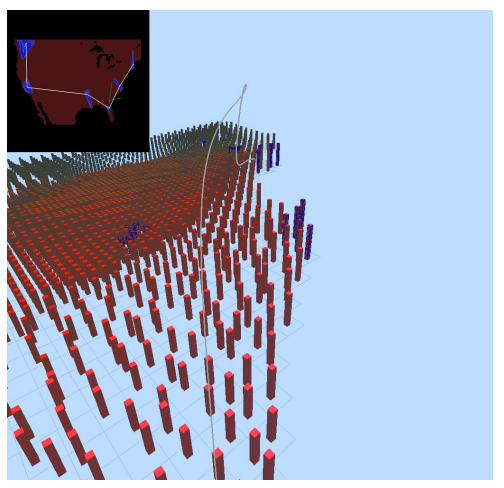

(h)

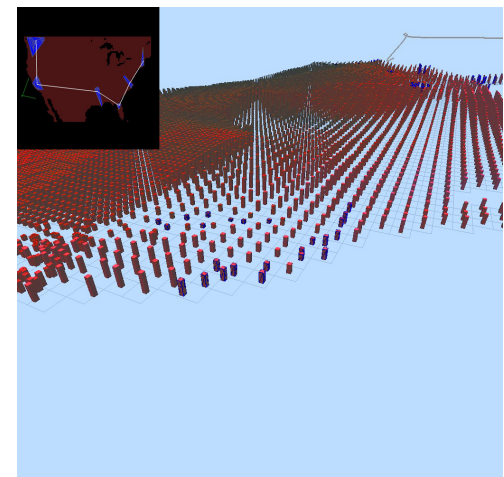

(c)

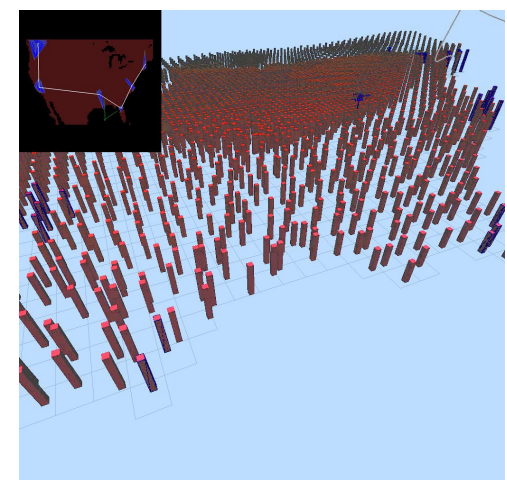

(f)

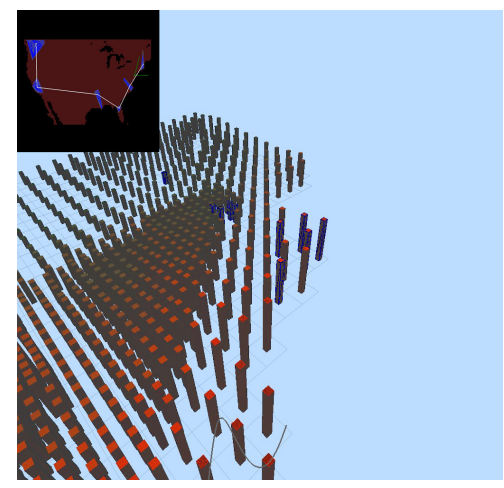

(i)

Figure 7.5: (a)-(c) depicts the camera moving from the Pacific Northwest to the Southwestern United States. (d)-(f) show the camera moving along the southern boundary of the United States towards Florida. (g)-(i) show the camera's path from Florida to the Eastern seaboard destination. 


\section{Chapter 8}

\section{Conclusions and Future Work}

As datasets continue to grow in size and dimensionality, scientists and engineers are forced to manage larger and more complex collections of data. It is rarely possible to successfully visualize the local details of an entire dataset in one unified view. Therefore, techniques are needed to assist users with locating interesting data and moving important offscreen information into view.

The navigation assistant described in this thesis attempts to address these issues by providing new techniques to help users successfully identify interesting data elements within a sea of visual information. It offers a structured framework for navigation assistance. The framework is made up of spatially coherent groups (AOIs) of elements of interest (EOIs). EOIs in the same AOI are connected with a local graph. AOIs are joined with a global graph. The graph framework provides visual information about the spatial relationships between EOIs belonging to the same AOI, and about how the AOIs are located throughout the dataset. The framework 
also acts as a data structure that is used to generate effective tours within the visualization. Graph algorithms and camera planning techniques are employed to build smooth animations to assist with data exploration.

Based on our successful results from using the navigation framework, future work is possible in a number of areas. First, additional types of tours that may provide new types of insight to a dataset should be explored. Consider a tour that circles an AOI. This might offer the user contextual information about the AOI's position in the dataset. Investigation is also needed to study the potential benefits of assigning different weight values to graph edges. Currently, the edge weights are the Euclidean distance between two vertices. In effect, these weights generate "short" tours. Assigning other meaningful weights to a graph edge might produce other types of useful animations. For example, touring from one EOI to another may force a relaxation of the user's preferences. It might be possible to avoid using edges that violate the user's constraints by increasing their weights to reflect a penalty for their use. It is still unclear, however, exactly how to adjust edge weights to produce the desired results.

Work is needed to extend the guidelines for cinematography agents and camera planners. Our system is primarily concerned with aiding users during data exploration. Within this narrow scope, additional guidelines could be constructed to improve the exploration process. One possible improvement is to have the assistant provide a rich variety of contextual shots intermixed with detailed close-ups. The navigation assistant's constraint solver can be extended to help address such cinematographic issues. Additional constraints could be added to build more informative shots, e.g. by requiring subsequent EOIs to be included in the view of the EOI 
currently being visited.

Lastly, the navigation assistant relies on a user to explicitly identify elements of interest using the rule grammar. Research is needed to construct effective implicit indicators. Can the navigation assistant learn what makes a data element interesting to the user by studying how the user interactively controls the camera? If a user spends an extended period of time viewing a particular region, can learn-by-example techniques be used to accurately construct new rules from the properties of EOIs in this region? As implicit rules are constructed and the goals of a user change, the user may need the ability to edit or remove the implicit rules generated by the assistant.

The system in this thesis offers several improvements over existing visualization systems:

1. Multidimensional visualization techniques are used to visualize a dataset. An overview of this visualization is provided to assist users with navigating the dataset, and a local view is displayed that allows users to study details about individual data elements.

2. Visual cues about the spatial relationships of EOIs and AOIs are provided by the edges of both a global graph and a set of local graphs. These visual cues assist users with making correct navigation decisions.

3. Camera planning techniques are used to build automated camera tours based on user requests. These tours allow users to focus on visualizing their data without the burden of navigating the visualization.

In summary, the navigation assistant in this thesis combines information and scientific vi- 
sualization techniques with graph theory and camera planning to provide effective data exploration and analysis within a dataset. 


\section{Bibliography}

[AC91] Bowen Alpern and Larry Carter. Hyperbox. In Gregory M. Nielson and Larry Rosenblum, editors, Proceedings of IEEE Visualization '91, pages 133-139, San Diego, CA, October 1991.

[BE94] Marla J. Baker and Stephen G. Eick. Space-filling software visualization. Journal of Visal Languages and Computing, 6(2):119-133, 1994.

[Bed95] Benjamin B. Bederson. Pad++: A zoomable graphical sketchpad for exploring alternate interface physics. Journal of Visual Langugages and Computing, 7(1):331, 1995.

[BETT99] Giuseppe Di Battista, Peter Eades, Roberto Tamassia, and Ioannis G. Tollis. Graph Drawing: Algorithms for the Visualization of Graphs. Prentice Hall, Upper Saddle River, NJ, 1999.

[BF01] Richard L. Burton and J. Douglas Faires. Numerical Analysis. Brooks/Cole, Pacific Grove, CA, seventh edition, 2001.

[BH94] Benjamin B. Bederson and James D. Hollan. Pad++: A zooming graphical interface for exploring alternate interface physics. In Proceedings of UIST '94, pages 17-26, New York, 1994. ACM.

[BHDH95] Lyn Bartram, Albert Ho, John Dill, and Frank Henigman. The continuous zoom: A constrained fisheye technique for viewing and navigating large information spaces. In Proceedings of UIST '95, pages 207-215, New York, 1995. ACM.

[BL99] William H. Bares and James C. Lester. Intelligent mult-shot visualization interfaces for dynamic 3d worlds. In Proceedings of IUI '99, pages 119-126, New York, 1999. ACM.

[BRT95] Lawrence D. Bergman, Bernice E. Rogowitz, and Lloyd A. Treinish. A rule-based tool for assisting colormap selection. In Proceedings Visualization '95, pages 118125, Atlanta, 1995.

[CIP96] Matthew Chalmers, Robert Ingram, and Christoph Pfranger. Adding imageability features to information displays. In Proceedings of UIST '96, pages 33-39, New York, 1996. ACM. 
[CK99] Ward Cheney and David Kincaid. Numerical Mathematics and Computing. Brooks/Cole, Pacific Grove, CA, 4th edition, 1999.

[CMS99] Stuart K. Card, Jock D. Mackinalay, and Ben Shneiderman. Readings in Information Visualization : Using Vision to Think. Morgan Kaufmann, San Francisco, 1999.

[DMR90] A. Dillon, C. McKnight, and J. Richardson. Navigation in hypertext : a critial review of the concept. In Proceedings INTERACT'90, pages 587-592, 1990.

[DS73] Roger M. Downs and David Stea. Cognitive maps and spatial behavior : Process and products. In Image and Environment. Aldine, Chicago, roger m. downs and david stea edition, 1973.

[DS93] Rudolph P. Darken and John L. Sibert. A toolset for navigation in virtual environments. In ACM User Interface Software and Technology, pages 157-165, 1993.

[DS96] Rudolph P. Darken and John L. Sibert. Wayfinding Strategies and Behaviors in Large Virtual Worlds. In Human Factors in Computing Systems: Proceedings of CHI'96, pages 142-149, New York, 1996. ACM.

[DZ94] Steven M. Drucker and David Zeltzer. Intelligent camera control in a virtual environment. Graphics Interface '94, pages 190-198, 1994.

[Ebe01] David H. Eberly. 3D Game Engine Design : A Practical Approach to Real-Time Computer Graphics. Morgan Kaufmann, San Francisco, CA, 2001.

[FB95] George W. Furnas and Benjamin B. Bederson. Space-scale diagrams : Understanding multiscale interfaces. In Proceedings of CHI'95, ACM Conference on Human Factors and Computing Systems, pages 234-241, New York, 1995. ACM.

[Fur81] George W. Furnas. The fisheye view: A new look at structured files. Technical report, AT \& T Bell Laboratories, Murray Hill, NJ, 1981.

[Fur86] George W. Furnas. Generalized fisheye views. In Proceedings of CHI'86 ACM Conference on Human Factors and Computing Systems, pages 16-23, New York, 1986. ACM.

[GFG $\left.{ }^{+} 94\right]$ N.D. Gershon, R.M. Friedhoff, J. Grass, R. Langridge, H.P. Meinzer, and J.D. Pearlman. Is visualization really necessary? the role of visualization in science, engineering and medicine. 21st Annual Conference on Computer Graphics and Interactive Techniques, 10(1):499-500, 1994.

[GGJ76] M. R. Garey, R. L. Graham, and D. S. Johnson. Some np-complete geometric problems. In Eighth Annual Symposium on Theory of Computing, pages 10-22, May 1976. 
[GPW89] Georges Grinstein, Robert M. Pickett, and Marian G. Williams. Exvis: An exploratory data visualization environment. In Proceedings of Graphics Interface '93, pages 254-261, London, Canada, 1989.

[HE98] Christopher G. Healey and James T. Enns. Building perceptual textures to visualize multidimensional datasets. In Proceedings of IEEE Visualization '98, pages 111118, Research Triangle Park, North Carolina, 1998.

[HE99] Christoher G. Healey and James T. Enns. Large datasets at a glance: Combining textures and colors in scientific visualization. IEEE Transactions on Visualization and Computer Graphics, 5(2):145-167, April-June 1999.

[IB95] Rob Ingram and Steven Benford. Legibility enhancement for information visualization. In Proceedings of IEEE Visualization '95, pages 209-216, 1995.

[JB97] Liqun Jin and David C. Banks. Tenisviewer: A browser for competition trees. IEEE Computer Graphics and Applications, pages 63-65, July/August 1997.

[JF97] Susanne Jul and George W. Furnas. Navigation in electronic worlds, 1997.

[Jul81] Bela Julesz. Textons, the elements of texture perception, and their interactions. Nature, 290:91-97, 1981.

[KL88] B.J. Kuipers and T.S. Levitt. Navigation and mapping in large-scale space. AI Magazine, 9(2):25-43, 1988.

[KPS97] Harsha P. Kumar, Catherine Plaisant, and Ben Shneiderman. Browsing hierarchical data with multi-level dynamic queries and pruning. International Journal of Human-Computer Studies, 46(1):103-124, 1997.

[Lyn60] Kevin Lynch. Image of the City. MIT Press, Cambridge, Massachusetts, 1960.

[MDB87] Bruce H. McCormick, Thomas A. Defanti, and M. D. Brown. Visualization is scientific computing. Computer Graphics, 21(6):1-14, 1987.

[MDR93] Cliff McKnight, Andrew Dillon, and John Richardson. Space-the final chapter or why physical representations are not semantic intentions. In Hypertext : A Psychological Perspective. Ellis Horwood, New York, cliff mcknight, andrew dillon, and john richardson edition, 1993.

[MF95] Sougata Mukherjea and James D. Foley. Showing the context of nodes in the world-wide web. In Human Factors in Computing Systems: Proceedings of CHI'95(Companion Volume, pages 326-327, New York, 1995. ACM.

[Mod97] David Modjeska. Navigation in electronic worlds : A research review. Technical report, University of Toronto, 1997. 
[MRC91] Jock D. Mackinlay, George G. Robertson, and Stuart K. Card. The perspective wall: Detail and context smoothly integrated. In Proceedings of CHI'91, ACM Conference of Human Factors in Computing Systems, pages 173-179, New York, April 1991. 1991.

[Noi93] Emanuel G. Noik. Layout-independent fisheye views of nested graphs. In Proceedings of 1993 IEEE Symposium of Visual Languages, pages 336-341, Bergen, 1993.

[Pap77] C. H. Papadimitriou. The euclidean traveling salesman problem is np-complete. Theoretical Computer Science, 4:237-244, 1977.

[Pas84] Remedi Passini. Wayfinding in Architecture. Van Nostrand Reinhold, New York, 1984.

[PG88] Ronald M. Pickett and Georges G. Grinstein. Iconographic displays for multidimensional data. In Proceedings of IEEE Conference on System, Man, and Cybernetics, pages 514-519, Beijing and Shenyang, PRC, 1988.

[PMR ${ }^{+96]}$ Catherine Plaisant, Brett Milash, Anne Rose, Seth Widoff, and Ben Shneidermann. Lifelines : Visualizing personal histories. In Proceedings of CHI'96, ACM Conference of Human Factors in Computing Systems, pages 221-227, New York, 1996. ACM.

[PS76] C. H. Papadimitriou and K. Steiglitz. Some complexity results for the travaling salesman problem. In Eighth ACM Annual Symposium on Theory of Computing, pages 1-9, May 1976.

[PS85] Franco P. Preparata and Michael Ian Shamos. Computational Geometry : An Introduction. Springer-Verlag, New York, 1985.

[RC94] Ramana Rao and Stuart K. Card. The table lens: Merging graphical and symbolic representations in an interactive focus+context visualization for tabular information. In Proceedings of CHI'94, ACM Conference of Human Factors in Computing Systems, pages 318-322, New York, 1994. ACM.

[RM92] George G. Robertson and Jock D. Mackinlay. The document lens. In Proceedings of the ACM Symposium on User Interface Software and Technology, pages 101108, New York, 1992. ACM.

[RMC91] George G. Robertson, Jock D. Mackinlay, and Stuart K. Card. Cone trees : Animated 3d visualizations of hierarchical information. In Proceedings of CHI'91, ACM Conference on Human Factors in Computing Systems, pages 189-194, New York, 1991. ACM.

[RSL77] D. J. Rosenkrantz, R. E. Stearns, and P. M. Lewis. An analysis of several heuristics for the traveling salesman problem. SIAM Journal of Computing, 6:563-581, 1977. 
[SA82] Robert Spence and Mark Apperley. Data base navigation: an office environment for the professional. Behavior and Information Technology, 1(1):43-54, 1982.

[SB92] Manojit Sarkar and Marc H. Brown. Graphical fisheye views of graphs. In Proceedings of CHI'92, ACM Conference on Human Factors in Computing Systems, pages 83-91, New York, 1992. ACM.

[Shn92] Ben Shneiderman. Tree visualization with tree-maps : 2-d space-filling approach. ACM Transactions on Graphics, 11(1):92-99, January 1992.

[Shu90] Simon B. Shum. Real and virtual spaces : mapping from spatial cognition to hypertext. In Hypermedia. Taylor and Graham, London, 1990.

[SP73] Stevens and P.Coupe. Distortions in judged spatial relations. Cognitive Psychology, 1973.

[Spe99] Robert Spence. A framework for navigation. Int. J. Human-Computer Studies, 51(5):919-945, November 1999.

[SSTR93] Manojit Sarkar, Scott S. Snibbe, Oren J. Tversky, and Steven P. Reiss. Stretching the rubber sheet: A metaphor for viewing large layouts on small screens. In Proceedings of the ACM Symposium on User Interface Software and Technology, pages 81-91. ACM, 1993.

[SZG ${ }^{+}$96] Doug Schaffer, Zhengping Zuo, Saul Greenberg, Lyn Bartram, John Dill, Shelli Dubs, and Mark Roseman. Navigating hierarchically clustered networks through fisheye and full zoom methods. ACM Transactions on Computer-Human Interaction, 3(2):162-188, June 1996.

[THR82] Perry W. Thorndyke and Barbara Hayes-Roth. Differences in spatial knowledge acquired from maps and navigation. In Cognitive Psychology. Academic Press, New York, 1982.

[Tol48] Edward C. Tolman. Cognitive maps in ratgs and men. The Psychological Review, 1948.

[vWvL93] Jarke J. van Wijk and Robert van Liere. Hyperslice. In Gregory M. Nielson and Daniel Bergeron, editors, Proceedings IEEE Visualization '93, pages 119-125, San Jose, California, October 1993.

[War88] Colin Ware. Color sequences for univariate maps: Theory, experiments, and principles. IEEE Computer Graphics and Applications, 8(5):41-49, 1988.

[WB97] Pak Chung Wong and R. Daniel Bergeron. 30 years of multidimensional multivariate visualization. Scientific Visualization, pages 3-33, 1997. 
[Wes01] Douglas B. West. Introduction to Graph Theory. Prentice-Hall, Upper Saddle River, NJ, 2nd edition, 2001.

[wHCS96] Li wei He, Michael F. Cohen, and David H. Salesin. The virtual cinematographer : A paradigm for automatic real-time camera control and directing. In SIGGRAPH '96, pages 217-223, 1996.

[WK95] Colin Ware and William Knight. Using visual texture for information display. Transactions on Graphics, 13(1):3-20, January 1995. 